\title{
$1 \quad$ Piezo1 activation using Yoda1 inhibits macropinocytosis and proliferation of cancer cells
}

2 Masashi Kuriyama ${ }^{1, \#}$, Hisaaki Hirose ${ }^{1, \#, *}$, Toshihiro Masuda ${ }^{1}$, Masachika Shudou ${ }^{2}$, Jan Vincent

3 V. Arafiles ${ }^{1}$, Miki Imanishi ${ }^{1}$, Masashi Maekawa ${ }^{3,4}$, Yuji Hara ${ }^{5, \dagger}$ and Shiroh Futaki ${ }^{1, *}$

$4 \quad{ }^{1}$ Institute for Chemical Research, Kyoto University, Uji, Kyoto 611-0011, Japan

$5 \quad{ }^{2}$ Division of Analytical Bio-Medicine, Advanced Research Support Center, Ehime University,

6 Toon, Ehime 791-0295, Japan.

$7 \quad{ }^{3}$ Division of Cell Growth and Tumor Regulation, Proteo-Science Center, Ehime University,

8 Toon, Ehime 791-0295, Japan.

$9 \quad{ }^{4}$ Department of Biochemistry and Molecular Genetics, Ehime University Graduate School of

10 Medicine, Toon, Ehime 791-0295, Japan.

$11{ }^{5}$ Department of Synthetic Chemistry and Biological Chemistry, Graduate School of 12 Engineering, Kyoto University, Katsura, Kyoto 615-8510, Japan.

13 †resent address: School of Pharmaceutical Sciences, University of Shizuoka, Shizuoka 422-

148526, Japan

15 \#These authors equally contributed to this work.

*Correspondence: hirose.hisaaki.5m@kyoto-u.ac.jp, futaki@scl.kyoto-u.ac.jp

\section{Keywords}




\section{Abstract}

Macropinocytosis is a type of endocytosis accompanied by actin rearrangement-driven membrane deformation, such as lamellipodia formation and membrane ruffling, followed by macropinosome formation. A certain number of mammalian mechanosensors are sensitive to membrane deformation and tension. However, it remains unclear whether macropinocytosis is regulated by mechanosensors. Focusing on the mechanosensitive ion channel Piezo1, we found that Yoda1, a Piezo1 agonist, potently inhibits macropinocytosis induced by epidermal growth factor (EGF). Although studies with Piezo1 knockout cells suggest that Piezo1 itself is not physiologically indispensable for macropinocytosis regulation, Yodal inhibited ruffle

30 formation depending on the extracellular $\mathrm{Ca}^{2+}$ influx through Piezol and on the activation of the calcium-activated potassium channel $\mathrm{KCa} 3.1$. This suggests that $\mathrm{Ca}^{2+}$ ions can regulate EGF-stimulated macropinocytosis. Moreover, Yoda1 impaired cancer cell proliferation, suggesting the impact of macropinocytosis inhibition. We propose the potential for cancer therapy by macropinocytosis inhibition through the regulation of a mechanosensitive channel activity. 


\section{Introduction}

Macropinocytosis is a large-scale endocytic pathway that accompanies membrane ruffling driven by actin rearrangement, followed by ruffle closure to form a vesicle called macropinosome $(0.2-10 \mu \mathrm{m} \text { in diameter })^{1-3}$. Macropinosomes are significantly larger than vesicles produced by other endocytic pathways ( $100 \mathrm{~nm}$ in diameter), such as clathrinmediated endocytosis, and macropinocytosis can non-selectively engulf a large volume of extracellular medium containing amino acids and proteins ${ }^{3,4}$. Moreover, it has been shown that macropinocytosis plays a key role in promoting the survival of cancer cells through nonselective uptake of extracellular proteins and nutrients ${ }^{5-7}$, suggesting that the development of macropinocytosis inhibitors could be applicable for cancer therapy. From the viewpoint of the intracellular delivery research field, macropinocytosis has been applied and manipulated as an efficient internalization route for the uptake of macromolecules such as antibodies, proteins, and drugs ${ }^{8,9}$. Therefore, understanding the molecular mechanisms of macropinocytosis and finding a new method to control this pathway should provide critical and useful insights not only for the development of cancer drugs, but also for drug delivery strategies.

The molecular mechanism of macropinocytosis has been studied in various types of cells, and there are two distinct forms of macropinocytosis: constitutive macropinocytosis and stimulated macropinocytosis ${ }^{10}$. Some specific cell types such as macrophages, dendritic cells, and Rastransformed cancer cells constantly perform macropinocytosis without a stimulus. Recent studies have elucidated the molecular mechanisms underlying constitutive macropinocytosis. For instance, Canton et al. have reported that constitutive macropinocytosis in macrophages is dependent on calcium-sensing receptors (CaSR) and extracellular calcium ions $\left(\mathrm{Ca}^{2+}\right)^{11}$. In contrast, macropinocytosis can be induced by various stimulants. For example, growth factors such as epidermal growth factor (EGF) and platelet-derived growth factor (PDGF), chemokines 
such as stromal cell-derived factor $1 \alpha$ (SDF1 $\alpha$, also known as CXCL12), and chemical compounds such as phorbol 12-myristate 13-acetate (PMA) can induce macropinocytosis in a wide range of cell types ${ }^{12-15}$. Notably, the molecular mechanisms by which EGF induces macropinocytosis have been extensively studied. EGF binds to the EGF receptor (EGFR) to activate small GTPases such as Racl and phosphatidylinositol 3-kinase (PI3K), leading to actin rearrangement and sequential conversion of phosphoinositides (i.e., phosphorylation and dephosphorylation), followed by dynamic membrane ruffling, ruffle closure, and formation of macropinosomes ${ }^{16}$.

In addition to further progress in understanding growth factor-stimulated macropinocytosis ${ }^{15}$, a recent study reported that a decrease in plasma membrane tension could induce macropinocytosis ${ }^{17}$, implying that alteration in membrane tension may regulate macropinocytosis. Membrane tension has been suggested to significantly impact on various cellular functions ${ }^{18}$. A previous study showed that membrane tension regulates cell spreading and lamellipodial extension ${ }^{19}$. Eukaryotic cells have various mechanosensors that sense and respond to membrane tension ${ }^{20,21}$. Mechanosensors respond to mechanical stimuli, such as alterations in membrane tension, to trigger signaling cascades that result in physiological responses $^{22}$. To the best of our knowledge, there are no reports on the relationship between mechanosensors and macropinocytosis regulation.

Among mechanosensors, mechanosensitive ion channels that couple mechanical force with ion flux have been extensively studied to elucidate their physiological roles and gating mechanisms ${ }^{21}$. In particular, much attention has been paid to the recently identified Piezo1, a mammalian mechanosensitive ion channel ${ }^{23}$. Piezo1 is a non-selective cation channel for $\mathrm{Na}^{+}$, $\mathrm{K}^{+}$, and $\mathrm{Ca}^{2+}$, with a higher preference for $\mathrm{Ca}^{2+}$ over $\mathrm{Na}^{+}$and $\mathrm{K}^{+}$ions ${ }^{23}$. Piezol has been reported to play key roles in a plethora of physiological phenomena such as flow sensing in 
endothelial cells, bone formation, myotube formation, $\mathrm{T}$ cell activation and regulation of red blood cell volume ${ }^{24-28}$. Recent studies have suggested that Piezo1 is involved in the regulation of actin polymerization ${ }^{29,30}$. In addition, Piezo1 is activated by mechanical stimuli as well as a small chemical compound called Yoda1, which was discovered by a high-throughput screen to activate Piezo1 ${ }^{31}$. Yoda1 is a known Piezol-specific agonist and is generally used to activate Piezo $1^{32}$.

Since macropinocytosis is accompanied by membrane deformation and actin rearrangements, macropinocytosis may affect membrane tension and the activities of mechanosensitive channels or vice versa. The above studies and insights concerning Piezo1 motivated us to focus on the role of Piezo1 in EGF-stimulated macropinocytosis. In addition, it remains unclear whether $\mathrm{Ca}^{2+}$ ions affect the regulation of EGF-stimulated macropinocytosis. Therefore, we investigated the effect of Piezol activation using Yoda1 and extracellular $\mathrm{Ca}^{2+}$ influx on macropinocytosis. Here we show that Yoda1 potently inhibits EGF-stimulated macropinocytosis and that Yoda1 impairs cancer cell proliferation. This work suggests that Yodal enhances $\mathrm{Ca}^{2+}$ influx followed by aberrant activation of the calcium-activated potassium channel $\mathrm{KCa} 3.1$ and inhibition of Rac1 activation. We propose that $\mathrm{Ca}^{2+}$ is a of key factor in the regulation of EGF-stimulated macropinocytosis. Importantly, our study suggests the potential for cancer therapy by inhibiting macropinocytosis by regulating a mechanosensitive channel.

\section{Results}

\section{Piezo1 activation using Yoda1 inhibits EGF-stimulated macropinocytosis in A431 cells.}

We used the human epidermoid carcinoma cell line A431, which expresses high levels of the EGFR and is a representative cell line for research on EGF-stimulated macropinocytosis. A previous study has reported that EGF-stimulated macropinocytosis in A431 cells can be 
evaluated by the amount of cellular uptake of tetramethylrhodamine (TMR)-conjugated dextran $70 \mathrm{kDa}$ (TMR-dex70), a macropinosome marker, using flow cytometry and confocal microscopy analysis ${ }^{33}$.

To assess whether Piezol activation is involved in the regulation of EGF-stimulated macropinocytosis in A431 cells, we used Yoda1. A431 cells were incubated with TMR-dex70 in the presence or absence of EGF and Yoda1, and cellular uptake of TMR-dex70 was quantitatively evaluated by flow cytometry and observed by confocal microscopy. EGF treatment enhanced the cellular uptake of TMR-dex70, whereas Yoda1 significantly inhibited the EGF-induced uptake of TMR-dex70 in a concentration-dependent manner (Figure 1A, B), indicating that Yoda1 $(1.5 \mu \mathrm{M})$ almost completely inhibited the cellular uptake of TMR-dex70 induced by EGF. On the other hand, Yoda1 did not substantively inhibit ( $\sim 10 \%$ inhibition at most) the clathrin-dependent cellular uptake of Alexa Fluor 568-labeled Transferrin (Tfn) into A431 cells (Figure 1-figure supplement 1). These results suggest that Piezo1 activation using Yoda1 selectively inhibits EGF-stimulated macropinocytosis.

To determine whether the inhibitory effect of Yoda1 on macropinocytosis is Piezo1-dependent, we established Piezo1 knockout (KO) A431 cells, using the CRISPR/Cas9 system $^{34}$. We obtained two clones (clones \#1 and \#2) in which Yoda1-induced $\mathrm{Ca}^{2+}$ influx, which can be detected by fluorescence of GCaMP6s, a genetically encoded $\mathrm{Ca}^{2+}$ indicator ${ }^{35}$, was completely abolished (Figure 1C). We also confirmed that ionomycin, a $\mathrm{Ca}^{2+}$ ionophore, induced $\mathrm{Ca}^{2+}$ influx in both wild-type and Piezo1 KO cells, indicating that Yoda1-induced $\mathrm{Ca}^{2+}$ is dependent on Piezo1 (Figure 1C). The DNA sequences around the CRISPR/Cas9-targeting region in these two clones were further confirmed by genome DNA sequencing, and all the detected alleles in clones \#1 and \#2 had CRISPR/Cas9-mediated gene mutations were as follows: clone \#1 had 10, 16, and 33 bp-deletion mutations, and clone \#2 had 9 and 10-bp deletion mutations (Figure 
1-figure supplement 2A). Since there were alleles without frameshift mutations in both clones (i.e., 33 and 9-bp deletions, resulting in 11 and 3-amino acid deletions of Piezol protein, referred to as $\Delta 946-956$ and $\Delta 944-946$, respectively), we investigated whether these two

134 mutants lost the function of Piezo1. Piezo1 is a cation channel that allows $\mathrm{Ca}^{2+}$ influx $^{23}$.

135 Therefore, we monitored the fluorescence intensities of the $\mathrm{Ca}^{2+}$ indicator Fluo- 8 in the absence or presence of Yoda1 in HEK293T cells transiently expressing full-length Piezo1, $\Delta 946-956$, or $\Delta 944-946$ (Figure 1-figure supplement 2B). The mutant Piezo1( $\Delta 946-956)$ derived from the clone \#1 allele slightly induced $\mathrm{Ca}^{2+}$ influx in the presence of Yoda1, whereas the other mutant Piezo1( $\Delta 944-946)$ derived from the clone \#2 allele did not. Therefore, we used clone

$140 \quad \# 2$ as Piezo1 KO A431 cells for the subsequent experiments. Piezo1 gene expression in A431 wild-type (WT) and Piezo1 KO cells was further confirmed by real-time quantitative PCR (qPCR) (Figure 1-figure supplement 3). The results indicated that the mRNA level of the Piezol coding region was decreased by over $90 \%$ in the Piezo1 KO cell line, suggesting that

144 there is minimal expression of Piezo1( $(4944-946)$ in the Piezo1 KO cells. Using the Piezo1 KO 145 A431 cells, we conducted a dextran uptake assay. Flow cytometry analysis and confocal microscopy observation revealed that EGF induced macropinocytosis in Piezo1 KO A431 cells in both the absence and presence of Yoda1 (Figure 1D, E). These results show that Piezo1 itself is not physiologically required for EGF-stimulated macropinocytosis because macropinocytosis can be induced in Piezo1 KO A431 cells; however, the Piezo1 agonist Yodal inhibits macropinocytosis in a Piezo1-dependent manner.

Piezo1 activation inhibits peripheral ruffle formation by inhibition of Rac1 activation.

152 Macropinocytosis is an actin-driven, non-specific endocytosis process accompanied by the 153 following steps: 1) formation of membrane ruffles induced by actin reorganization and 2) 154 subsequent closure of the ruffles to form macropinosomes ${ }^{36}$. We sought to determine which step 
Yoda1 inhibits. We investigated peripheral ruffle formation by time-lapse live cell imaging and the membrane ${ }^{37}$. In the presence of Yoda1, the extension and folding back of the plasma membrane of A431 cells after EGF addition were clearly observed within 6 min. However, this phenomenon was absent in the presence of Yoda1 (Figure 2A and Video 1, 2). In addition, staining actin filaments using phalloidin revealed that Yodal inhibited actin polymerization. A431 cells were stimulated with EGF for $5 \mathrm{~min}$ in the presence or absence of Yodal and then fixed, followed by staining with rhodamine-phalloidin to detect F-actin; then, the cells with the F-actin positive peripheral ruffles were quantified (Figure 2B). EGF stimulation resulted in $\sim 25 \%$ of cells with peripheral ruffles, whereas co-treatment with Yoda1 significantly decreased the proportion of cells with peripheral ruffles $(\sim 4 \%)$. Moreover, scanning electron microscopy clearly showed that Yodal inhibited EGF-induced peripheral ruffle formation (Figure 2C).

We then investigated whether Yodal inhibited Racl activation. In the process of actin rearrangement to form membrane ruffles, EGF-induced actin rearrangement is due to the activation of the small GTPase Rac1 ${ }^{38}$. The pulldown experiment of active Rac1 (Rac1-GTP) showed that the amount of Rac1-GTP in the cells treated with EGF increased as previously reported $^{39}$. However, Yodal inhibited EGF-induced increase in the amount of Rac1-GTP (Figure 2D). These results show that Yodal inhibits Rac1 activation.

We examined three possibilities how Yodal affected Rac1. First, we investigated whether Yoda1 lowers cytosolic $\mathrm{pH}$. It has been previously shown that macropinocytosis inhibition by amiloride, an inhibitor of $\mathrm{Na}^{+} / \mathrm{H}^{+}$exchangers (NHE), is due to lower submembranous $\mathrm{pH}$, which prevents Rac1 activation ${ }^{39}$. As previously described, we used the dual-emission ratio (645/585 $\mathrm{nm}$ ) of seminaphthorhodafluor dye-5 (SNARF-5F) to quantify intracellular pH (Figure 2- 
with dimethyl sulfoxide (DMSO) as vehicle, Yoda1, or ethyl-isopropyl amiloride (EIPA), an amiloride derivative that is widely used as a macropinocytosis inhibitor. EIPA significantly decreased in cytosolic $\mathrm{pH}$, whereas Yoda1 did not lower cytosolic $\mathrm{pH}$ (Figure 2-figure supplement 1B), suggesting that Rac1 inhibition by Yodal is unlikely due to a decrease in intracellular $\mathrm{pH}$. Second, we examined the possibility of inhibition of EGF signal transduction leading to Rac1 activation. Yoda1 did not inhibit EGF-related signaling pathways such as phosphorylation of EGFR and Vav2 (Figure 2-figure supplement 2), suggesting that Yoda1 does not affect the acute response of phosphorylation induced by EGF signaling. Finally, we also checked whether Yoda1 affects cholesterol (Chol) distribution in cells. Because membrane ruffling and macropinocytosis in A431 cells require cholesterol to regulate the localization of Rac1 ${ }^{40}$, we investigated Chol distribution in the absence or presence of Yoda1 using a genetically encoded biosensor for Chol (mCherry-D4H) ${ }^{41}$. Time-lapse imaging showed that Yoda1 does not affect Chol distribution in the cells, suggesting that inhibition of macropinocytosis by using Yoda1 is not due to change in Chol localization (Figure 2-figure supplement 3).

Although the mechanism by which Yoda1 inhibits Rac1 activation remains unclear, these results show that Yodal inhibits EGF-induced actin reorganization and peripheral ruffle formation.

\section{Extracellular $\mathrm{Ca}^{2+}$ is required for macropinocytosis inhibition by Piezo1 activation.}

Since activated Piezol is known to be permeable to extracellular $\mathrm{Ca}^{2+}$ influx, we next examined whether extracellular $\mathrm{Ca}^{2+}$ influx is important for the inhibition of macropinocytosis by Piezo1 activation using Yoda1. To confirm the effects of Yoda1 on $\mathrm{Ca}^{2+}$ influx into A431 cells, we conducted time-lapse calcium imaging using A431 cells transiently expressing GCaMP6s. Yodal was added 8 min after time-lapse imaging started, resulting in an immediate increase in 
intracellular $\mathrm{Ca}^{2+}$ concentration (Figure 3A). Because Piezo1 and other mechanosensitive ion channels could be activated by shear stress such as stimulus by addition of buffer solution itself $\mathrm{f}^{27,42}$, Hanks' balanced salt solution (HBSS) containing DMSO was used as a vehicle control. After adding DMSO solution, the fluorescence of GCaMP6s did not increase, as shown in Fig. 3a, indicating that the intracellular $\mathrm{Ca}^{2+}$ concentration did not significantly increase. To investigate whether the intracellular calcium response induced by Yoda1 is due to extracellular $\mathrm{Ca}^{2+}$ influx, we used $\mathrm{Ca}^{2+}$-free HBSS. Under these conditions, Yoda1 did not increase intracellular $\mathrm{Ca}^{2+}$ concentrations (Figure 3A). These results indicate that Yodal causes extracellular $\mathrm{Ca}^{2+}$ influx but does not affect calcium release from intracellular $\mathrm{Ca}^{2+}$-storage organelles such as the endoplasmic reticulum.

We then investigated whether Yodal inhibits macropinocytosis also in $\mathrm{Ca}^{2+}$-free conditions.

213 The dextran uptake assay was conducted using a $\mathrm{Ca}^{2+}$-free medium. Yoda1 did not inhibit TMR214 dex70 uptake in the absence of extracellular $\mathrm{Ca}^{2+}$, indicating that extracellular $\mathrm{Ca}^{2+}$ influx 215 through Piezo1 is crucial for the inhibition of macropinocytosis by Yoda1 (Figure 3B, C). EGF216 induced uptake of TMR-dex70 in $\mathrm{Ca}^{2+}$-free medium without Yoda1 was significantly reduced 217 (by $\sim 40 \%$ ) compared to that in $\mathrm{Ca}^{2+}$-containing medium. EGF-stimulated macropinocytosis in 218 A431 cells has been previously reported to be independent of extracellular $\mathrm{Ca}^{2+}$ ion $^{11}$, but the 219 effect of extracellular $\mathrm{Ca}^{2+}$ may vary, likely due to differences in experimental conditions and 220 assay systems. However, this result indicates that Yoda1 did not inhibit EGF-stimulated 221 macropinocytosis under extracellular $\mathrm{Ca}^{2+}$-free conditions. These data suggest that Piezo1 222 activation by Yoda1 inhibits macropinocytosis in an extracellular $\mathrm{Ca}^{2+}$-dependent manner.

223 KCa3.1 activation is necessary for the inhibitory effect of Yoda1 on ruffle formation.

224 We then sought to identify molecule(s) that function downstream of Yoda1-induced $\mathrm{Ca}^{2+}$ 
signaling related to macropinocytosis inhibition. We focused on $\mathrm{KCa} 3.1$, a $\mathrm{Ca}^{2+}$-activated $\mathrm{K}^{+}$ channel that is activated by $\mathrm{Ca}^{2+}$ influx through Piezol and reduce cell volume in red blood cells $^{28}$. KCa3.1 also plays a key role in EGF-stimulated macropinocytosis ${ }^{33}$. In macropinocytosis, sequential dephosphorylation of phosphoinositides $\left(\mathrm{PI}(3,4,5) \mathrm{P}_{3} \rightarrow \mathrm{PI}(3,4) \mathrm{P}_{2}\right.$ $\rightarrow \mathrm{PI}(3) \mathrm{P} \rightarrow \mathrm{PI})$ is required ${ }^{43}$. KCa3.1 has been reported to be activated by $\mathrm{PI}(3) \mathrm{P}$ and is also crucial for macropinocytic cup formation ${ }^{33,44}$. Therefore, proper temporal activation of KCa3.1 at a later stage of the macropinocytosis process is required for completion of macropincytosis.

We hypothesize that Yoda1-induced $\mathrm{Ca}^{2+}$ influx acutely activates $\mathrm{KCa} 3.1$, and that the improper activation of KCa3.1 inhibits ruffle formation. Since inhibition of KCa3.1 impairs macropinosome formation but does not affect ruffle formation ${ }^{33}$, the involvement of $\mathrm{KCa} 3.1$ in the inhibition of macropinocytosis by Yoda1 was tested by a membrane ruffling assay. A431 cells were pretreated with TRAM-34, a potent and selective KCa3.1 inhibitor, and then treated with EGF and Yoda1 in the presence of TRAM-34. Live cell differential interference contrast (DIC) imaging and phalloidin staining showed that KCa3.1 inhibition by TRAM-34 restored EGF-induced peripheral ruffle formation in the presence of Yoda1 (yellow arrowheads, Figure

4A, B and Video 3). We also compared the effects of Yoda1 with ionomycin on the inhibition of macropinocytosis. Ionomycin treatment led to increased intracellular $\mathrm{Ca}^{2+}$ concentration (Figure. 1C), and it has been reported that ionomycin induces phospholipase C (PLC) activation to hydrolyze $\mathrm{PI}(4,5) \mathrm{P}_{2}$ into diacylglycerol (DAG) and inositol-3-phosphate $\left(\mathrm{IP}_{3}\right)^{45}$.

$244 \mathrm{PIP}_{2}$ breakdown is thought to lead to inhibition of macropinocytosis, because sequential 245 phosphorylation and dephosphorylation of $\mathrm{PIP}_{2}$ is required. Therefore, we checked the amount of $\mathrm{PI}(4,5) \mathrm{P}_{2}$ in the plasma membrane using a genetically encoded biosensor of $\mathrm{PI}(4,5) \mathrm{P}_{2}(\mathrm{GFP}-$ PLC $\delta$-PH $)^{46}$. Time-lapse imaging showed that ionomycin treatment led to complete redistribution of the $\mathrm{PIP}_{2}$ biosensor on the plasma membrane to the cytosol as previously 
reported ${ }^{45}$, whereas Yoda1 treatment did not induce the redistribution of the biosensor (Figure

4-figure supplement 1A). This suggests that Yodal treatment, unlike ionomycin, does not hydrolyze $\mathrm{PIP}_{2}$ despite increased intracellular $\mathrm{Ca}^{2+}$ concentration. In addition, A431 cells pretreated with TRAM-34 did not recover peripheral membrane ruffling in the presence of ionomycin during EGF stimulation (Figure 4-figure supplement 1B and Video 4). Altogether, these results suggest that the inhibitory effect of Yoda1 on EGF-stimulated macropinocytosis is related to $\mathrm{KCa} 3.1$ activation by an increase in $\mathrm{Ca}^{2+}$ concentration through Piezol activation; however, the mechanism by which Yodal inhibits macropinocytosis is completely different from that of ionomycin.

\section{Pharmacological activation of KCa3.1 inhibits EGF-induced macropinocytosis.}

To further confirm that KCa3.1 activation is involved in the inhibition of macropinocytosis, we next investigated whether the pharmacological activation of $\mathrm{KCa} 3.1$ by using SKA-31, a potent potassium channel activator, inhibits EGF-induced membrane ruffle formation and macropinocytic uptake. SKA-31 is known to activate $\mathrm{KCa} 3.1$ and $\mathrm{KCa}^{47}$. A431 cells were stimulated with EGF in the absence or presence of SKA-31, and a membrane ruffling assay was performed (Figure 4C). Although SKA-31 did not completely inhibit EGF-mediated membrane ruffle induction compared to the effect of Yoda1, there was a significant decrease in the formation of membrane ruffles induced by EGF. Inhibition of macropinocytosis by SKA-31 was also confirmed by flow cytometry analysis and confocal microscopy observation of TMRdex70 uptake (Figure 4D, E). SKA-31 significantly inhibited the uptake of TMR-dex70 by the 269 addition of EGF. Altogether, these results indicate that $\mathrm{KCa} 3.1$, if activated in a non-temporal 270 manner, can inhibit EGF-stimulated macropinocytosis.

271 Taken together with our findings in this study, it is suggested that Yoda1 specifically activates 
Piezo1, which leads to acute activation of $\mathrm{KCa} 3.1$, followed by inhibition of actin rearrangement due to preventing Rac1 activation (Figure 4F).

\section{Piezo1 activation using Yoda1 inhibits cancer cell proliferation.}

Macropinocytosis functions as a nutrient supply pathway in cancer cells ${ }^{5,48}$. Therefore, inhibition of macropinocytosis could be a target for a cancer therapy ${ }^{7,49}$. In this study, we further examined whether Yoda1 treatment for longer incubation (e.g., $16 \mathrm{~h})$ in serum-containing medium inhibits cellular uptake of TMR-dex70 as a marker of macropinocytosis in A431 cells and whether Yoda1 inhibits A431 cell proliferation. A431 cells were incubated with TMRdex70 and Yoda1 for $16 \mathrm{~h}$, and the cellular uptake of dextran in serum-containing medium was measured by flow cytometry analysis and microscopy observation (Figure 5A, B). Even under these experimental conditions, Yoda 1 reduced TMR-dex 70 uptake in a concentration-dependent manner. TMR-dex70 uptake in $16 \mathrm{~h}$ was also inhibited by EIPA, a representative inhibitor of macropinocytosis by inhibiting NHE, suggesting that macropinocytosis may contribute to dextran uptake in A431 cells for $16 \mathrm{~h}$ in serum-containing medium (Figure 5-figure supplement 1).

A cell proliferation assay using WST-8 formazan, colony formation assay, and wound healing assay were performed to investigate whether Piezo1 activation by Yoda1 inhibited A431 cell proliferation. The WST-8 assay showed that Yoda1 reduced cell proliferation in a concentrationdependent manner (Figure 5C). This inhibitory effect was completely abolished in Piezo1 KO A431 cells, demonstrating that decreased proliferation was mediated by Piezo1 activation (Figure 5C). Yodal treatment at $3 \mu \mathrm{M}$ showed the maximum effect in these experiments, and as such, we used Yoda1 at $3 \mu \mathrm{M}$ in the subsequent experiments. The colony formation assay also showed that Yodal inhibited long-term cell proliferation (i.e., 12 days) (Figure 5D). 
Furthermore, the wound healing assay indicated that cell proliferation and migration were inhibited by Piezo1 activation using Yoda1 (Figure 5E).

We next tested whether Yodal has an impact on Ras-transformed cancer cells in which macropinocytosis constantly occurs. Using HT1080 cells, a human fibrosarcoma NRas-mutant cell line, we examined the effect of Yoda1 on dextran uptake and cell proliferation. We confirmed Piezo1 gene expression in HT1080 cells by qPCR (Figure 5-figure supplement 2A). The dextran uptake assay showed that constitutive macropinocytosis in HT1080 cells was inhibited by Yoda1 in a concentration-dependent manner (Figure 5-figure supplement 2B, C). In addition, the wound-healing assay showed that proliferation and migration of HT1080 cells were inhibited in the presence of Yoda1, although the effect of inhibition of macropinocytosis and proliferation was not significant compared to that in A431 cells (Figure 5-figure supplement 2D). However, these data suggest that Yoda1 also has promising activity in the inhibition of constitutive macropinocytosis and cell proliferation in Ras-transformed cells. Altogether, these results suggest that inhibition of macropinocytic cellular uptake through Piezol activation leads to the inhibition of cancer cell proliferation.

\section{Discussion}

Our findings demonstrate that Piezo1 activation using Yoda1, a Piezo1 agonist, leads to potent inhibition of EGF-stimulated macropinocytosis in A431 cells. We demonstrated that Yoda1 strongly inhibited the formation of membrane peripheral ruffles induced by EGF. In addition, we also showed that Yoda1 has an inhibitory effect on constitutive macropinocytosis in HT1080 cells, a representative Ras-transformed cancer cell line. In contrast, in Piezo1 KO A431 cells, EGF-stimulated macropinocytosis was observed even in the presence of Yoda1. This result suggests that Piezo1 itself is not crucial for the physiological regulation of macropinocytosis 
processes that accompany membrane dynamics, such as membrane ruffling. In addition, this study used Yoda1. Although our results cannot strictly conclude that macropinocytosis can be regulated by Piezo1 activity through a physiological increase in membrane tension, we found that Yoda1 inhibits macropinocytosis depending on Piezo1.

We showed that the inhibitory effect of Yodal on macropinocytosis was dependent on extracellular $\mathrm{Ca}^{2+}$ influx (Figure 3). This indicates that $\mathrm{Ca}^{2+}$ influx through Piezo1 activation by Yoda1 is essential for the inhibition of macropinocytosis. The inhibition of KCa3.1, which is a calcium-activated potassium channel, recovered the formation of peripheral membrane ruffles in the presence of Yoda1 (Figure 4A, B). Therefore, we propose that acute KCa3.1 activation due to Piezo1 activation by Yodal likely leads to the inhibition of macropinocytosis. In addition, we showed that a $\mathrm{KCa} 3.1$ activator as well as Yodal also impaired macropinocytosis (Figure 4C, D, E). On the other hand, a previous study reported that KCa3.1 activation is essential to complete macropinosome formation, the later stage of macropinocytosis process ${ }^{33}$. Altogether, our results suggest that appropriate temporal activation of $\mathrm{KCa} 3.1$ is important in macropinocytosis.

Furthermore, we demonstrated that Yoda1 treatment inhibited the activation of Rac1, which is essential for membrane ruffling induced by actin rearrangement (Figure 2D). A previous study reported that knockdown of Piezo1 in gastric cancer cells led to Rac1 activation ${ }^{50}$. Taken together, our results suggest that Piezo1 activation inhibits Rac1 activation. However, as mentioned above, membrane ruffling was observed even in the presence of Yoda1 in the case of pretreatment with TRAM-34 (Figure 4A, B). This result suggests that Rac1 can be activated even in the presence of Yoda1 when $\mathrm{KCa} 3.1$ is inhibited. We propose that $\mathrm{KCa} 3.1$ activation, following Yoda1-induced Piezo1 activation, could lead to the inhibition of Rac1 activation

341 (Figure 4F). Further studies are required to elucidate the relationship between KCa3.1 and 
Rac1.

Macropinocytosis has recently attracted much more attention, especially from the point of view

344 of cancer metabolism ${ }^{5,51,52}$. Preventing macropinocytosis in cancer cells is thought to be an important method for cancer therapy ${ }^{49}$. Therefore, further understanding of the molecular mechanisms and physiological significance of macropinocytosis is required. Unfortunately, there are very few specific and versatile inhibitors for macropinocytosis, because few specific proteins and lipids related to macropinocytosis have been identified ${ }^{53}$. This makes it challenging to develop specific pharmacological tools to inhibit macropinocytosis ${ }^{54}$. Conventional macropinocytosis inhibitors such as cytochalasin D, wortmannin, and EIPA can affect other endocytic pathways or have off-target effects. For instance, cytochalasin D blocks not only macropinocytosis and affects receptor-mediated endocytosis ${ }^{55}$. Wortmannin blocks membrane ruffle closure of macropinocytosis by inhibiting PI3K ${ }^{56}$. Although wortmannin is considered to be a highly selective inhibitor of PI3K, it can also potently inhibit mammalian polo-like kinase 1 (PLK1), which is critical in mitosis ${ }^{57}$. EIPA is one of the most common reagents to inhibit macropinocytosis by blocking NHE, but it also blocks transient receptor potential polycystic 3 (TRPP3), a $\mathrm{Ca}^{2+}$-activated channel belonging to the TRP superfamily of cation channels ${ }^{58}$.

This study suggests the possibility of developing a new macropinocytosis inhibitor based on Yoda1. To the best of our knowledge, Yoda1 has so far been considered a highly specific reagent for Piezo1. Although some off-target effects of Yoda1 might exist ${ }^{59}$, our data indicate that Yoda1 inhibits macropinocytosis depending on Piezol activation and extracellular $\mathrm{Ca}^{2+}$ influx. Interestingly, the inhibitory mechanism of Yodal should be clearly different from that of $\mathrm{Ca}^{2+}$

363 elevation using ionomycin, the $\mathrm{Ca}^{2+}$ ionophore (Figure 4A and Figure 4-figure supplement leading to Piezol activation might also have the potential to inhibit macropinocytosis. 
From the viewpoint of the potential of Yoda1 as a cancer drug, we also demonstrated that Yoda1 dependent on the inhibition of macropinocytosis through Piezol activation (Figure 5B, C, D, E). These data suggest that Yoda1 and Piezo1 activation may be useful for preventing cancer cell proliferation. In contrast, other studies have suggested that Piezol has a key role in promoting cancer cell proliferation and tumor growth ${ }^{50,60,61}$. These studies imply that the effect of Piezol activation on cell proliferation may vary depending on cell types, and Yoda1 may inhibit the proliferation of specific cancer cells. In addition, methods for targeting cancer cells to activate Yodal are required, because Piezol has been reported to be involved in various physiological functions ${ }^{62}$, implying that there may be unexpected side effects in normal cells and tissues if Piezol is non-selectively activated. Further research is needed to clarify the factors that significantly affect cancer cell proliferation in the presence of Yoda1. inhibits EGF-stimulated macropinocytosis in A431 cells, which could lead to the inhibition of cell proliferation. Moreover, our results showed that extracellular $\mathrm{Ca}^{2+}$ influx through Piezo1 modulates EGF-stimulated macropinocytosis, suggesting the impact of $\mathrm{Ca}^{2+}$ on the regulation of macropinocytosis. This study paves the way for the development of novel drugs and methods for studying macropinocytosis study and for cancer therapy by regulating mechanosensitive channel activity.

\section{Materials and methods}

\section{Reagents}


reagents and culture media were also purchased either from FUJIFILM Wako Pure Chemical Corporation, Sigma-Aldrich, or Thermo Fisher Scientific, unless otherwise specified.

\section{Cell culture}

A431 and HT1080 cells were cultured in Dulbecco's modified Eagle's medium (D-MEM, high glucose) (FUJIFILM Wako Pure Chemical Corporation) supplemented with 10\% (v/v) heatinactivated fetal bovine serum (FBS) (Gibco) [D-MEM(+)]. HEK293T cells were cultured in D-MEM with low glucose (FUJIFILM Wako Pure Chemical Corporation) supplemented with $10 \%(\mathrm{v} / \mathrm{v})$ heat-inactivated FBS. All cells were maintained at $37{ }^{\circ} \mathrm{C}$ in a humidified $5 \% \mathrm{CO}_{2}$ incubator and passaged every 2-4 days. Cells were used for experiments between passage numbers 1 and 15 .

\section{Plasmids construction}

pGP-CMV-GCaMP6s-CAAX was a gift from Dr. Tobias Meyer (Addgene, \#52228) ${ }^{63}$. pGPCMV-GCaMP6s was generated by deleting the CAAX sequence from pGP-CMV-GCaMP6sCAAX (Addgene, \#52228). To construct the expression plasmid pIRES2-mCherry, cDNA encoding ZsGreen1 was removed from pIRES2-ZsGreen1 (Takara) by digestion with BstXI/NotI, and then an mCherry cDNA fragment was inserted into the same sites. pPiezo1IRES2-mCherry was generated by inserting human Piezol cDNA (Flexi ORF Clone \#FXC01061) into the EcoRI/BamHI sites of pIRES2-mCherry. pPiezo1( $\Delta 946-956)-I R E S 2-$

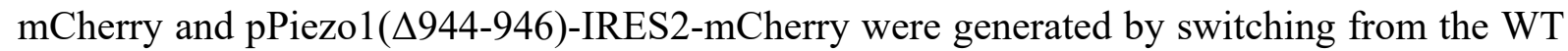
to the deleted sequences between the MluI and SalI sites of pPiezo1-IRES2-mCherry. The deleted sequences were generated as follows: two sequences for each deleted sequence were amplified from pPiezo1-IRES2-mCherry using the following pairs of primers: Piezo1 mutant forward 1 and Piezo1 mutant reverse1, Piezo1 mutant forward2 and Piezo1 mutant reverse2, 
Piezo1 mutant forward1 and Piezo1 mutant reverse3, and Piezo1 mutant forward3 and Piezo1 mutant reverse 2 . Then, the two oligos were ligated and digested using the primers forward 1 and reverse2 and MluI and SalI, respectively. pSpCas9(BB)-2A-Puro (PX459) V2.0 was a gift from Feng Zhang (Addgene plasmid \# 62988; http://n2t.net/addgene:62988; RRID: Addgene_62988) ${ }^{34}$. To construct the CRISPR-Cas9 plasmid for Piezo1-knockout (referred to as PX459-Piezo1), pSpCas9(BB)-2A-Puro (PX459) V2.0 was digested using BbsI-HF (NEB) at $37{ }^{\circ} \mathrm{C}$ for $60 \mathrm{~min}$, followed by deactivation at $65{ }^{\circ} \mathrm{C}$ for $20 \mathrm{~min}$. After cooling down, QuickCIP (NEB) was added and the mixture was further incubated at $37{ }^{\circ} \mathrm{C}$ for $10 \mathrm{~min}$, and then deactivated at $80{ }^{\circ} \mathrm{C}$ for $2 \mathrm{~min}$. The digested plasmid was purified using the Wizard SV

Gel and PCR Clean-up system (Promega).

A guide sequence (TATTCGAGGCCATCGTGTACCGG) to knock out human Piezo1 (accession number: NM_001142864.4) was determined using CRISPRdirect (https://crispr.dbcls.jp). Two oligos, oligo 1 and oligo 2, were phosphorylated using T4 PNK (NEB) and annealed to clone the guide sequence into the sgRNA scaffold of the plasmid. Ligation was then performed by combining the BbsI-digested PX459, the annealed oligo duplex at a 1:3 mol ratio and ligation mix (Takara Bio) at $16{ }^{\circ} \mathrm{C}$ for $30 \mathrm{~min}$. The ligation mixture was introduced into Escherichia coli DH5 $\alpha$, and the insert sequence was verified by standard sequencing.

\section{Transfection}

Transfection of plasmids was performed using Lipofectamine LTX (Invitrogen) according to the manufacturer's protocol. Plasmids, Lipofectamine LTX, and PLUS reagent were diluted in Opti-MEM (Invitrogen) and incubated at $25^{\circ} \mathrm{C}$ for $5 \mathrm{~min}$ for complex formation. The mixture was then added to each dish, resulting in a final plasmid concentration of $2.5 \mu \mathrm{g} / \mathrm{mL}$. The culture medium was changed $4 \mathrm{~h}$ after the transfection. Subsequent experiments were performed $24 \mathrm{~h}$ after transfection. 


\section{Establishment of Piezo1-KO A431 cell line}

437

438

439

440

A431 cells $\left(7 \times 10^{5}\right.$ cells/dish) were seeded onto a $60 \mathrm{~mm}$ dish (Iwaki) and incubated overnight. The cells were then transfected with the PX459-Piezo1 plasmid using Lipofectamine LTX (Thermo Fisher Scientific), according to the manufacturer's instructions, after which they were washed with PBS twice at $6 \mathrm{~h}$ after transfection and incubated in D-MEM(+) for 1 day. Afterwards, they were washed with PBS twice and incubated in D-MEM $(+)$ containing puromycin (1 $\mu \mathrm{g} / \mathrm{mL})$ (Sigma) for 3 days. Then the cells were washed twice with PBS and incubated with D-MEM(+) without puromycin for 3 days. Finally, the cells were collected and seeded onto a 96-well plate (Iwaki) by limiting dilution to isolate and culture single-cell clones.

\section{Sequencing of CRISPR/Cas9 target site of the Piezo1 gene}

Genome DNA was extracted from A431 cells (wild type, clones \#1 and \#2) using GeneArt Genomic Cleavage Detection Kit (Thermo Fisher Scientific), following the manufacturer's protocol. The sequence around the target of CRISPR/Cas9 was amplified using the following pair of primers: Piezo1 gDNA forward and Piezo1 gDNA reverse. The amplified product was purified using Wizard SV Gel and PCR Clean-up system (Promega) and inserted into T-Vector pMD20 (Takara Bio) using DNA Ligation Kit $<$ Mighty Mix $>$ (Takara Bio) according to the manufacturer's protocol. The ligation mixture was introduced into Escherichia coli DH5 $\alpha$ and the insert sequence was verified by standard sequencing.

\section{RT-PCR and real-time PCR.}

Total RNA was extracted from A431 cells using NucleoSpin RNA Plus (Takara Bio), following the manufacturer's protocol. The quantity and quality of RNA was measured by a nanodrop (Thermo Fisher Scientific). RNA concentration was determined by absorbance at $260 \mathrm{~nm}$ and 
RNA quality was confirmed by the $260 / 280 \mathrm{~nm}$ ratio. $2 \mu \mathrm{g}$ of total RNA was subsequently reverse transcribed to cDNA using PrimeScript RT Master Mix (Takara Bio) according to the manufacturer's protocol. RT-PCR was done using GoTaq Green Master Mix (Promega). The resulting DNA was fractionated by agarose gel electrophoresis (135 V, $20 \mathrm{~min})$ and viewed with UV transilluminator. Real-time PCR was performed using PowerUp SYBR Green Master Mix (Thermo Fisher Scientific) and 7300 Real-Time PCR System (Applied Biosystems). GAPDH was used as a reference gene.

\section{Dextran uptake assay}

Intracellular uptake of TMR-dex70 was evaluated using confocal microscopy observation and flow cytometry analysis. A431 cells $\left(2 \times 10^{5}\right.$ cells/dish and $1 \times 10^{5}$ cells/well $)$ were seeded onto $35 \mathrm{~mm}$ glass-bottomed dishes (Iwaki) and a 24-well plate (Iwaki), respectively, and incubated for 1 day. The cells were washed with PBS twice and cultured in D-MEM(-) overnight for serum-starvation. The starved A431 cells were treated with TMR-dex70 $(0.5 \mathrm{mg} / \mathrm{mL})$ and reagents as indicated on corresponding figure legends for $10 \mathrm{~min}$ at $37{ }^{\circ} \mathrm{C}$. For confocal microscopy observation, the cells were then washed twice with ice-cold PBS and stained nuclei with Hoechst $33342(5 \mu \mathrm{g} / \mathrm{mL}$, Invitrogen) for $10 \mathrm{~min}$. The observation was carried out using an FV1000 confocal laser scanning microscope (CLSM) system (Olympus) equipped with a $60 \times$ objective lens (UPlanSApo, oil immersion, NA 1.35; Olympus). For flow cytometry analysis, the cells were washed twice with ice-cold PBS, detached from the plate with $0.25 \%$ trypsin in PBS for 10 min at $37^{\circ} \mathrm{C}$, added D-MEM(+) to prevent further digestion, and collected into centrifuge tubes. The cells were then centrifuged $\left(800 \times g, 5 \mathrm{~min}, 4{ }^{\circ} \mathrm{C}\right)$ and the resulting pellets were washed with ice-cold PBS. The cells were centrifuged again, washed with ice-cold PBS once more and filtrated with a cell strainer. Flow cytometry analysis was performed with 10,000 gated events using an Attune NxT Flow Cytometer (Thermo Fisher Scientific). The 
results are shown as relative median fluorescence intensity of 10,000 counted events. For HT1080 cells, experiments were conducted without serum starvation. HT1080 cells $\left(2 \times 10^{5}\right.$ cells/dish and $6 \times 10^{4}$ cells/well) were seeded onto $35 \mathrm{~mm}$ glass-bottomed dishes and a 24 -well plate, respectively, and incubated for 1 day. The cells were incubated with TMR-dex70 (0.5 $\mathrm{mg} / \mathrm{mL})$ in the absence or presence of Yodal in D-MEM(+) for $4 \mathrm{~h}$ at $37^{\circ} \mathrm{C}$ and then subjected to confocal microscopy observation and flow cytometry analysis as described above.

\section{Tfn uptake assay}

A431 cells $\left(2 \times 10^{5}\right.$ cells/dish and $1 \times 10^{5}$ cells/well $)$ were seeded onto $35 \mathrm{~mm}$ glass-bottomed dishes and a 24-well plate and incubated for 1 day, and then serum-starved in D-MEM(-) for 1 $\mathrm{h}$ prior to experiments. The cells were incubated with AF568-Tfn $(20 \mu \mathrm{g} / \mathrm{mL})$ in the absence or presence of Yoda1 $(1.5 \mu \mathrm{M})$ in D-MEM(-) for 10 min at $37^{\circ} \mathrm{C}$. The cells were then acidwashed twice with Glycine-HCl buffer (with $150 \mathrm{mM} \mathrm{NaCl}, \mathrm{pH} 3.0$ ) to remove AF568-Tfn on the plasma membrane. Then the cells were fixed with 4\% PFA in case of confocal microscopy observation. Microscopy observation and flow cytometry analysis of cellular uptake of Tfn were performed as described above in the method for dextran uptake assay.

\section{Time-lapse live cell imaging}

A431 cells $\left(2 \times 10^{5}\right.$ cells/dish) were seeded onto $35-\mathrm{mm}$ glass-bottomed dishes (Iwaki) and incubated for 1 day. If necessary, the cells were transfected with the indicated plasmids and serum-starved prior to the experiments. The cells were washed twice with PBS, and the culture medium was replaced with D-MEM(-) $(150 \mu \mathrm{L}$, on the glass part of the dish). The cells were placed at $37^{\circ} \mathrm{C}$ in a microchamber (STXG-IX3WX-SET; Tokai Hit) attached on the FV3000 microscope stage. Reagents in D-MEM(-) $(50 \mu \mathrm{L})$ were added to the cells to yield the final concentrations indicated in the corresponding figure legends. DIC and fluorescence images 
were captured every 10 or 20 s using an FV3000 confocal laser scanning microscope (CLSM) system (Olympus) equipped with a $60 \times$ objective lens (UPlanSApo, oil immersion, NA 1.35; Olympus).

\section{$\mathrm{Ca}^{2+}$ imaging using GCaMP6s}

A431 cells $\left(2 \times 10^{5}\right.$ cells/dish) seeded onto $35-\mathrm{mm}$ glass-bottomed dishes (Iwaki) were transfected with a plasmid to express GCaMP6s. The cells were washed with Hanks' balanced salt solution (HBSS; $400 \mathrm{mg} / \mathrm{L} \mathrm{KCl}, 60 \mathrm{mg} / \mathrm{L} \mathrm{KH}_{2} \mathrm{PO}_{4}, 8,000 \mathrm{mg} / \mathrm{L} \mathrm{NaCl}, 350 \mathrm{mg} / \mathrm{L} \mathrm{NaHCO}_{3}$, $60 \mathrm{mg} / \mathrm{L} \mathrm{Na}_{2} \mathrm{HPO}_{4} \cdot \mathrm{H}_{2} \mathrm{O}, 1,000 \mathrm{mg} / \mathrm{L}$ D-Glucose, containing $1 \mathrm{mM} \mathrm{Ca}^{2+}, 1 \mathrm{mM} \mathrm{Mg}^{2+}$ and $20 \mathrm{mM}$ HEPES at $\mathrm{pH} 7.4)$ and the culture medium was replaced with HBSS $(150 \mu \mathrm{L}$, on the glass part of the dish). GCaMP6s fluorescence images were acquired every 1 min as described above in the method for time-lapse live cell imaging. Fluorescence intensity was measured using ImageJ software (NIH). Yoda1-induced $\mathrm{Ca}^{2+}$ influx was quantified as the difference in the GCaMP6s fluorescence intensity between its maximum value $\left(\mathrm{F}_{\max }\right)$ and the basal level $\left(\mathrm{F}_{0}\right)$.

\section{$\mathrm{Ca}^{2+}$ imaging using Fluo-8 in HEK293T expressing Piezo1 WT or mutants}

HEK293T cells $\left(2 \times 10^{5}\right.$ cells/dish) were seeded onto $35 \mathrm{~mm}$ glass-bottomed dishes and transfected with either Piezol(full length)-IRES-mCherry, Piezo1( $\Delta 946-956)-I R E S-m C h e r r y$, Piezo1(4944-946)-IRES-mCherry or empty vector (IRES-mCherry). HEK293T cells expressing mCherry were considered to express Piezo1 WT or the mutants. The cells were treated with Fluo-8 AM (5 $\mu \mathrm{M}$, AAT Bioquest) for 45 min and then washed twice with PBS. The cells were then washed with Hanks' balanced salt solution (HBSS; $400 \mathrm{mg} / \mathrm{L} \mathrm{KCl,} 60$ mg/L $\mathrm{KH}_{2} \mathrm{PO}_{4}, 8,000 \mathrm{mg} / \mathrm{L} \mathrm{NaCl}, 350 \mathrm{mg} / \mathrm{L} \mathrm{NaHCO} 3,60 \mathrm{mg} / \mathrm{L} \mathrm{Na} 2 \mathrm{HPO}_{4} \cdot \mathrm{H}_{2} \mathrm{O}, 1,000 \mathrm{mg} / \mathrm{L}$ DGlucose, containing $1 \mathrm{mM} \mathrm{Ca}^{2+}, 1 \mathrm{mM} \mathrm{Mg}^{2+}$ and $20 \mathrm{mM}$ HEPES at pH7.4) and the culture medium was replaced with HBSS ( $150 \mu \mathrm{L}$, on the glass part of the dish). Fluo-8 fluorescence 
images were acquired every $1 \mathrm{~min}$ as described above in the method for time-lapse live cell imaging. Fluorescence intensity was measured using ImageJ software (NIH). Yoda1-induced $\mathrm{Ca}^{2+}$ influx was quantified as the difference in the Fluo-8 fluorescence intensity between its maximum value $\left(\mathrm{F}_{\max }\right)$ and the basal level $\left(\mathrm{F}_{0}\right)$.

\section{Membrane ruffling assay}

A431 cells $\left(2.5 \times 10^{5}\right.$ cells/dish $)$ were seeded onto $35 \mathrm{~mm}$ glass-bottomed dishes, cultured for 1 day and then serum-starved overnight prior to experiments. The cells were treated with reagents for 5 min at $37^{\circ} \mathrm{C}$, fixed with $4 \%$ paraformaldehyde in PBS for 10 min, and permeabilized with $0.1 \%$ Triton X-100 in PBS for 4 min. The cells were then incubated with $1 \%$ BSA in PBS for 30 min to block non-specific binding prior to staining F-actin with rhodamine-conjugated phalloidin (Invitrogen) for $30 \mathrm{~min}$. The observations were carried out using the FV1000 CLSM system (Olympus) equipped with a 40× objective lens (UPlanSApo, NA 0.95; Olympus). Among the cells at the margin of the colony, the cells with over $12 \mu \mathrm{m}$ accumulation of phalloidin were counted as cells with ruffles.

\section{Scanning electron microscopy}

A431 cells were stimulated with EGF $(20 \mathrm{nM})$ in the absence or presence of Yoda1 $(1.5 \mu \mathrm{M})$ for 5 min and then fixed with $2 \%$ glutaraldehyde and $4 \%$ paraformaldehyde in $0.1 \mathrm{M}$ cacodylate buffer ( $\mathrm{pH}$ 7.4) for $2 \mathrm{~h}$, washed with cacodylate buffer, and post-fixed with $1 \%$ osmium tetroxide in cacodylate buffer for $2 \mathrm{~h}$. After washing with distilled water, the specimens were subjected to the conductive staining with $1 \%$ buffered osmium tetroxide and $1 \%$ tannic acid (O-T-O methods). The specimens were then dehydrated in a graded series of ethanol and critical-point drying (Hitachi, Ltd. Critical Point Dryer HCP-1), coated with a thin layer (3 nm) of osmium coater (Vacuum Device Inc.; HPC-30W), and then observed with a field-emission 
scanning electron microscope (Hitachi S-4800, Tokyo, Japan) at $2 \mathrm{kV}$ acceleration voltage.

\section{Rac1 activation assay}

The Rac1 activation assay was conducted using the Rac1 Pull-Down Activation Assay Biochem experiments. The cells were treated with reagents for 3 min at $37^{\circ} \mathrm{C}$, lysed with $240 \mu \mathrm{L}$ of icetagged PAK-PBD beads. After washing the beads, bound proteins were eluted with SDSloading buffer. The lysates were applied into a polyacrylamide gel (SuperSep Ace, 5-20\%, 13well; FUJIFILM Wako Pure Chemical Corporation) and fractionated by SDS-PAGE. The (TBST) for $1 \mathrm{~h}$ at $25{ }^{\circ} \mathrm{C}$, and then incubated overnight at $4{ }^{\circ} \mathrm{C}$ with appropriate primary antibodies in 3\% skim milk in TBST. After washing the membrane with TBST for 10 min three times, the blots were further incubated with appropriate horseradish peroxidase (HRP)-

571 prime (GE Healthcare) and LAS3000 mini (FUJIFILM). The images were analyzed using 572 ImageJ software (NIH).

\section{Western blot}


A431 cells $\left(3 \times 10^{5}\right.$ cells/well) were seeded on a 6 -well plate (Iwaki), cultured for 1 day and serum-starved overnight prior to experiments. The cells were treated with EGF in the presence or absence of Yoda 1 for $5 \mathrm{~min}$ at $37^{\circ} \mathrm{C}$, lysed with ice-cold RIPA buffer [ $50 \mathrm{mM}$ Tris- $\mathrm{HCl}(\mathrm{pH}$ 7.6), $150 \mathrm{mM} \mathrm{NaCl}, 1 \mathrm{mM}$ EDTA, $1 \%$ Triton X-100, 0.1\% SDS, $0.1 \%$ sodium deoxycholate] containing protease inhibitor cocktails (Roche) and phosphatase inhibitor cocktails (Roche), and lysates were cleared by centrifugation at $16000 \times g$ for $20 \mathrm{~min}$ at $4{ }^{\circ} \mathrm{C}$. The protein concentrations of the lysates were measured by BCA protein assay using Pierce BCA Protein Assay Kit (Thermo Fisher Scientific) and then unified to $0.5 \mu \mathrm{g} / \mu \mathrm{L}$. SDS-PAGE, antibody treatment and detection were performed described as above in the method for Rac1 activation assay using 5\% BSA in TBST as blocking solution. When detecting EGFR and Vav2, the membrane was subjected for the first immunoblots (pEGFR or pVav2), stripped by immerging the membrane in Restore PLUS Western Blot Stripping Buffer (Thermo Fischer Scientific) for $5 \mathrm{~min}$ and washed twice with TBST for $10 \mathrm{~min}$ and then subjected to the second immunoblots (EGFR or Vav2).

\section{Intracellular pH measurement}

A431 $\left(2.5 \times 10^{5}\right.$ cells/dish) cells were seeded on $35 \mathrm{~mm}$ glass-bottomed dishes (Iwaki) and incubated for 1 day. The cells were incubated with SNARF-5F AM (20 $\mu \mathrm{M}$, Invitrogen) in D$\operatorname{MEM}(-)$ for 30 min at $37^{\circ} \mathrm{C}$, washed twice with PBS, and then incubated with Yoda1 $(1.5 \mu \mathrm{M})$ in D-MEM(-) for $10 \mathrm{~min}$ at $37^{\circ} \mathrm{C}$. EIPA $\left(25 \mu \mathrm{M}, 30 \mathrm{~min}, 37^{\circ} \mathrm{C}\right)$ was used as a positive control which decreases intracellular $\mathrm{pH}$. The observation was done using the FV1000 CLSM system (Olympus) equipped with a 40× objective lens (UPlanSApo, NA 0.95; Olympus). SNARF-5F AM was excited at $559 \mathrm{~nm}$ and images were acquired in the range of 570-600 $\mathrm{nm}$ and $630-660$ $\mathrm{nm}$ to evaluate emission signal at $585 \mathrm{~nm}$ and $645 \mathrm{~nm}$, respectively. Fluorescence intensity was measured using ImageJ software $(\mathrm{NIH})$ and ratio of emission signal of 585/645 nm was 
calculated. For establishing calibration curve, cells were incubated with SNARF-5F AM as above, washed twice with calibration buffer $\left(130 \mathrm{mM} \mathrm{KCl}, 10 \mathrm{mM} \mathrm{NaCl}, 1 \mathrm{mM} \mathrm{MgSO}_{4}, 10\right.$ $\mathrm{mM}$ MOPS) at $\mathrm{pH} 6.2,6.6,7.0,7.4,7.8$, or 8.2 , and then incubated with calibration buffer containing $10 \mu \mathrm{g} / \mathrm{mL}$ nigericin for $15 \mathrm{~min}$ at $25^{\circ} \mathrm{C}$ to equilibrate the intracellular $\mathrm{pH}$ with extracellular $\mathrm{pH}$.

\section{WST-8 assay}

The WST-8 assay was performed using the Cell Counting Kit-8 (Dojindo), according to the manufacturer's protocol. A431 cells $\left(1 \times 10^{4}\right.$ cells/well) were seeded onto a 96 -well plate (Iwaki) and incubated for 1 day. The cells were treated with DMSO or Yoda1 $(1.5,3$, or $6 \mu \mathrm{M})$ in D$\operatorname{MEM}(+)$ for $24 \mathrm{~h}$ at $37^{\circ} \mathrm{C}$. The cells were then washed twice with PBS before adding $100 \mu \mathrm{L}$ D-MEM(-) and $10 \mu \mathrm{L}$ of WST-8 reagent to each well. The cells were further incubated for $2 \mathrm{~h}$ at $37^{\circ} \mathrm{C}$ followed by measuring the absorbance at $450 \mathrm{~nm}$.

\section{Colony formation assay}

A431 cells $\left(4 \times 10^{2}\right.$ cells/dish) were seeded onto $35 \mathrm{~mm}$ dishes and incubated for 1 day. The cells were treated with DMSO or Yodal $(3 \mu \mathrm{M})$ in D-MEM(+) for 12 days at $37^{\circ} \mathrm{C}$, until the DMSOtreated cells formed visible colonies. The culture medium was changed every 3 days. The cells were then fixed with $4 \%$ paraformaldehyde for $20 \mathrm{~min}$ and stained with $0.2 \%$ crystal violet for $20 \mathrm{~min}$. The dishes were photographed, and visible colonies were counted.

\section{Wound healing assay}

A431 cells $\left(4 \times 10^{5}\right.$ cells $/$ dish $)$ were seeded onto $35 \mathrm{~mm}$ dishes and incubated for 2 days. A confluent monolayer of A431 cells was scratched with a $10 \mu \mathrm{L}$ pipette tip to create a scratch wound. The cells were then treated with DMSO or Yoda1 $(3 \mu \mathrm{M})$ in D-MEM(+) for $40 \mathrm{~h}$ at 
620

621

622

623

624

625

626

627

628

629

630

631

632

633

634

635

636

637

638

639

640

641

$37^{\circ} \mathrm{C}$. Phase contrast images of the wounded areas at 0,24 and $40 \mathrm{~h}$ after scratching were acquired using a CK40 inverted microscope (Olympus) equipped with a $4 \times$ objective lens (SPlan 4PL, NA 0.13; Olympus) and a 10× eyepiece lens (NCWHK; Olympus). The wounded areas were measured using ImageJ software (NIH).

\section{Statistical analysis}

No statistical method was used to determine the sample size prior to the study. All data are presented as the mean \pm standard error of the mean (SEM) of three independent biological replicates $(n=3)$ unless otherwise specified. All statistical analyses were performed using JMP Pro 14. For comparison of two groups, an unpaired Student's t-test was used. For multiple comparison analyses, one-way analysis of variance (ANOVA) followed by Tukey-Kramer's post hoc test or Dunnett's post hoc test was used. The calculated P-values were considered significant at $\mathrm{P}<0.05$.

\section{Acknowledgements}

pGFP-C1-PLC $\delta$-PH and pmCherry-C1-D4H were kind gifts from Dr. Gregory Fairn (St. Michael's Hospital, Toronto, Canada) ${ }^{41}$. This work was supported by JST CREST (Grant Number JPMJCR18H5).

\section{Author contributions}

H.H. conceived the project, designed the experiments, established the Piezo1 knockout A431 cells with the help of Y.H., and analyzed the data. M.K. designed and performed most experiments and analyzed the data. T.M., J.V.V.A., and M.I. contributed to analysis for flow cytometry, Rac1 pulldown assay, and plasmid construction for Piezo1 mutants, respectively. M.S. and M.M. performed the scanning electron microscopy observations. Y.H. contributed to 
642 construction of Piezo1-related plasmids and establishment of the Piezo1 knockout cell line.

643 M.K. and H.H. wrote the original manuscript. S.F. supervised the project and wrote the

644 manuscript with H.H. and M.K.. All authors discussed and commented on the manuscript.

\section{Competing interests}

646 The authors declare no competing interests. 
A

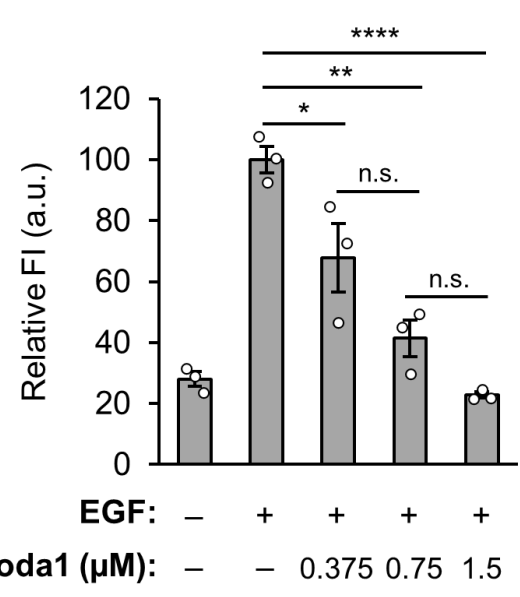

C

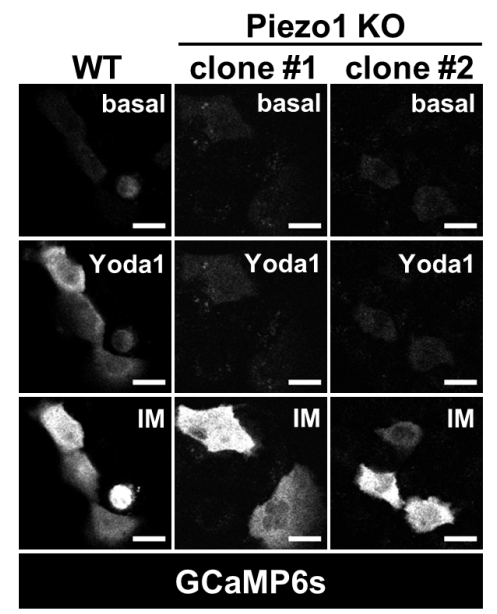

D

Piezo1 KO A431 cells (clone \#2)

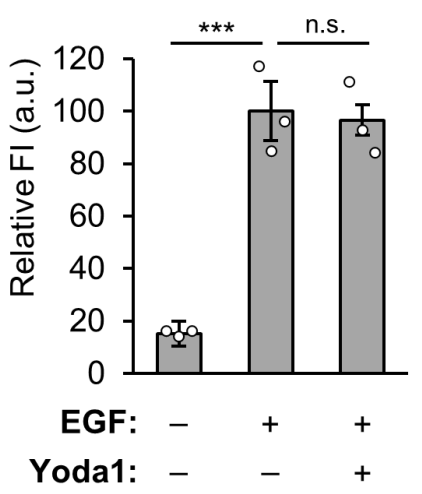

B
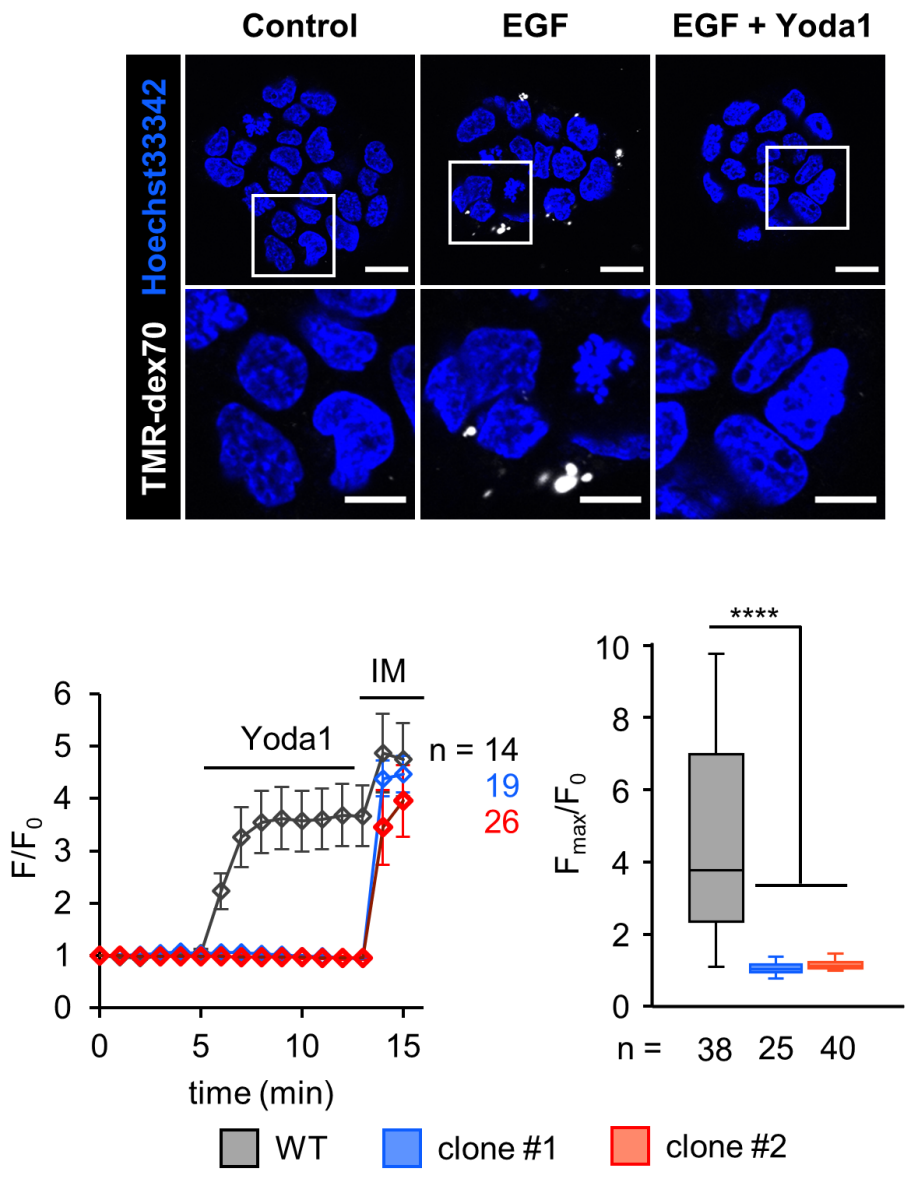

$\mathbf{E}$ Piezo1 KO A431 cells (clone \#2)

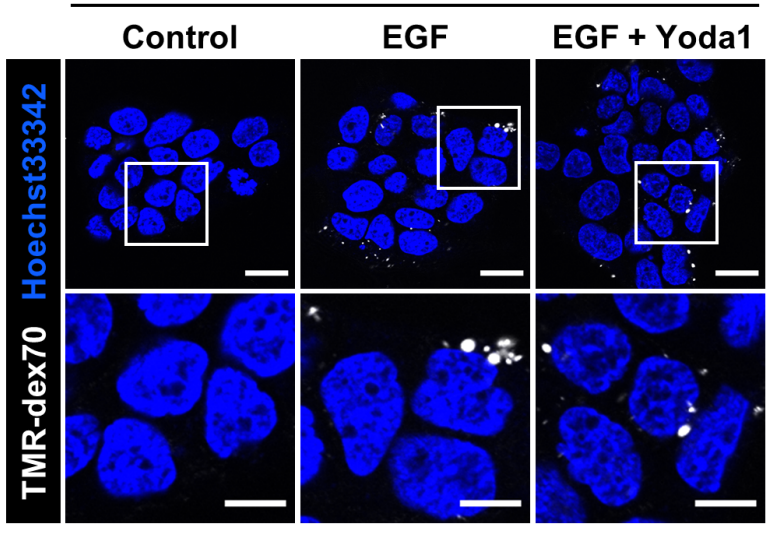

649 Figure 1. Piezo1 activation using Yoda1 inhibits macropinocytosis induced by EGF in A431

650 cells. (A) Flow cytometry analysis of TMR-dex70 uptake into A431 cells with or without EGF

$651(20 \mathrm{nM})$ in the absence or presence of Yodal at the indicated concentration for $10 \mathrm{~min}$. (B) 
Confocal microscopy observation of TMR-dex70 uptake into the cells stimulated with EGF (20 in WT, Piezo1 KO clone\#1 and clone \#2 cells were recorded every 1 min. Yoda1 (1.5 $\mu \mathrm{M})$ and ionomycin $(5 \mu \mathrm{M})$ were added at 5 and 13 min after start of time-lapse imaging, respectively. Left: Representative images of GCaMP6s-expressing cells before and after addition of Yoda1 and ionomycin. Middle: Representative time-course of relative fluorescence intensity of GCaMP6s. Right: Quantification of maximum Yoda1-induced GCaMP6s intensity increase. Box and whiskers graph: line, median; box, upper and lower quartiles; whiskers, maxima and minima. (D) Flow cytometry analysis of TMR-dex70 uptake into Piezo1 KO A431 cells (clone top images. Data represent the mean $\pm \operatorname{SEM}(n=3$ independent biological replicates in $(\mathbf{A})$ and (D); $\mathrm{n}=14,19,26$ cells for WT, clone \#1 and \#2, respectively) in (C middle)). Data represent in box plot (from left to right, $\mathrm{n}=38,25,40$ cells pooled from two independent experiments) in $\left(\mathbf{C}\right.$ right). ${ }^{*}, \mathrm{p}<0.05 ; * *, \mathrm{p}<0.01 ; * * *, \mathrm{p}<0.001 ; * * * *, \mathrm{p}<0.0001 ;$ n.s., not significant

671 Figure supplement 1. Tfn uptake in the absence or presence of Yoda1.

672 Figure supplement 2. Supporting data for characterization of Piezo1 knockout A431 cells.

673 Figure supplement 3. Piezo1 gene expression examined by qPCR. 
A
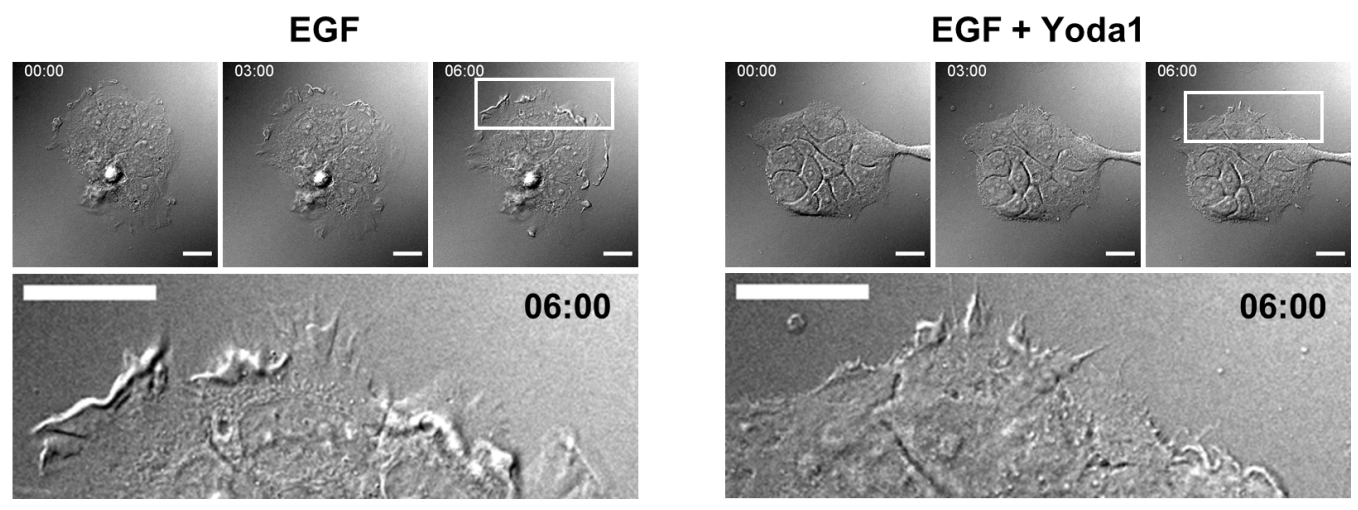

B
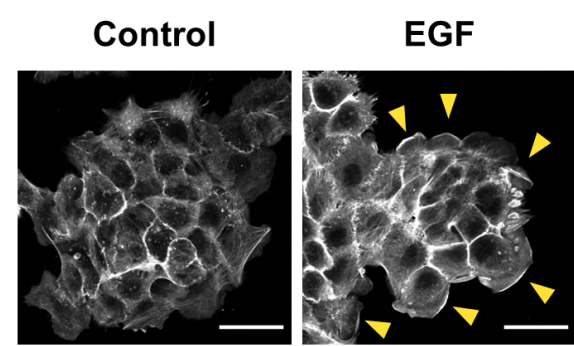

phalloidin

C
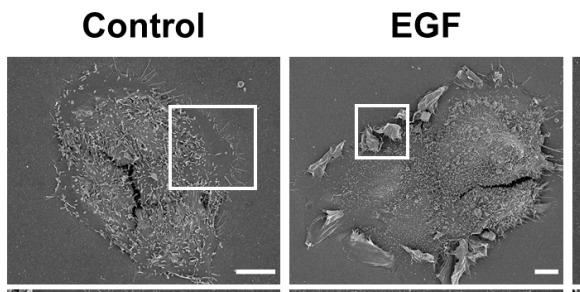

EGF + Yoda1
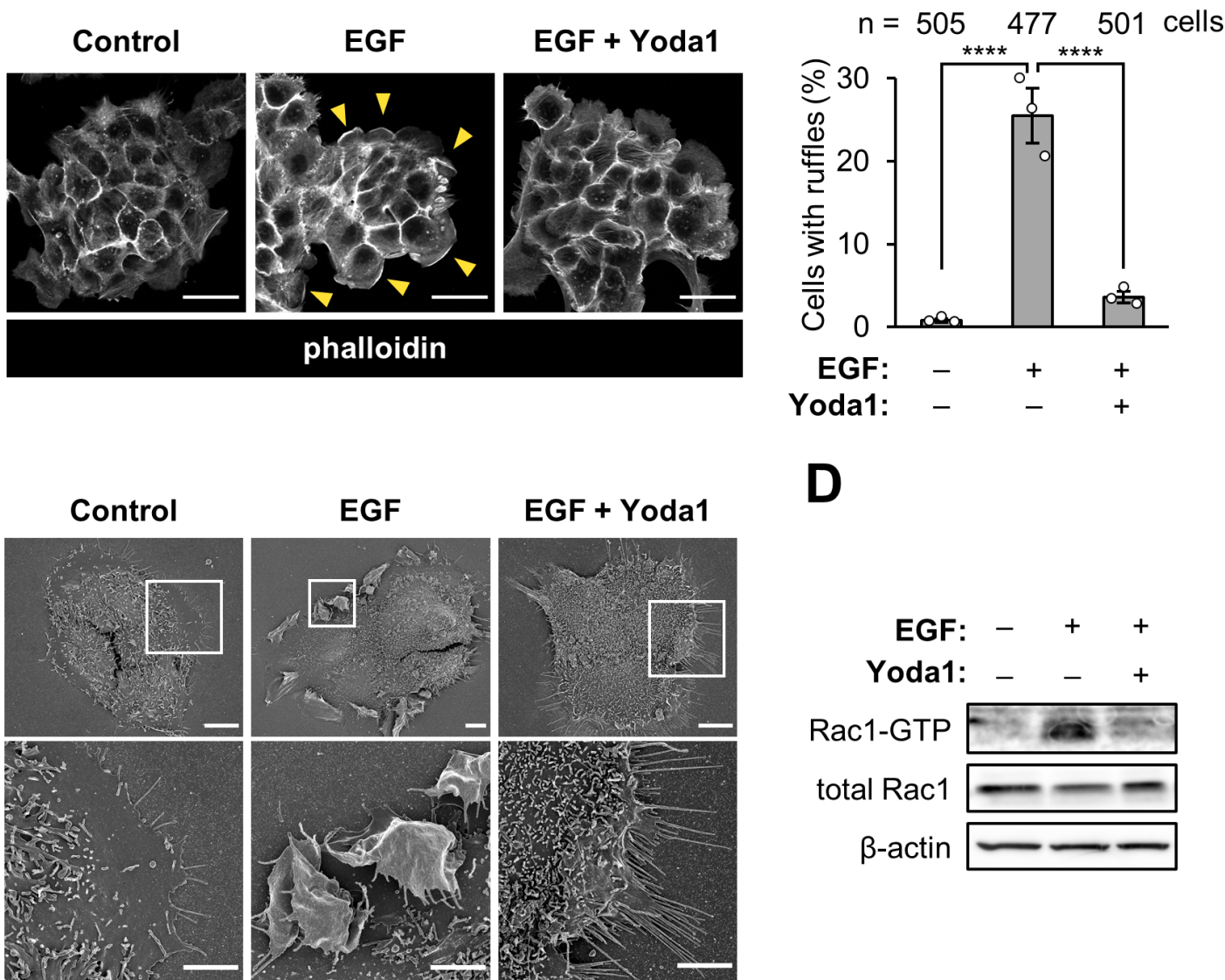

675

676 Figure 2. Piezo1 activation inhibits peripheral ruffle formation. (A) Live cell imaging of EGF-

677 induced membrane ruffles in A431 cells. The cells were stimulated with EGF (20 nM) in the

678 absence or presence of Yoda1 $(1.5 \mu \mathrm{M})$. Live cell imaging was started immediately after adding

679 EGF and Yoda1. Differential interference contrast (DIC) images at the indicated time points $(0$, 
680

681

682

683

684

685

686

687

688

689

690

691

692

693

694

695

696

697

698

3 and 6 min) are shown. The bottom images show enlarged views of the areas outlined by the white squares in the images at 6 min. (B) F-actin staining with phalloidin. A431 cells were stimulated with EGF $(20 \mathrm{nM})$ in the absence or presence of Yodal $(1.5 \mu \mathrm{M})$ for $5 \mathrm{~min}$, fixed, and stained with phalloidin-rhodamine to detect F-actin. Left: Representative images are shown. Yellow arrowheads indicate peripheral membrane ruffling area. Right: Quantification of cells with peripheral ruffles. Data represent the mean \pm SEM (number of total counted cells pooled from three independent experiments: from left to right, 505, 477, and 501). (C) Scanning electron microscopy images of A431 cells stimulated with EGF $(20 \mathrm{nM})$ in the absence or presence of Yoda1 $(1.5 \mu \mathrm{M})$ for $5 \mathrm{~min}$. The bottom images show enlarged views of the areas outlined by the white squares in the top images. (D) Rac1 activation pull-down assay. A431 cells treated with EGF $(20 \mathrm{nM})$ and Yoda1 $(1.5 \mu \mathrm{M})$ for 3 min were lysed and active Rac1 was precipitated using PAK-PBD beads. Active and total Rac1 protein were analyzed by performing SDS-PAGE followed by western blot. The representative images are shown. The experiments were performed twice with similar results. ****, $\mathrm{P}<0.0001$ (one-way ANOVA followed by Tukey-Kramer's post hoc test (B)). Scale bars, (A) $20 \mu \mathrm{m}$; (B) $50 \mu \mathrm{m}$; (C top) $10 \mu \mathrm{m}$; (C bottom) $5 \mu \mathrm{m}$.

Figure supplement 1. Yodal does not change intracellular $\mathrm{pH}$.

Figure supplement 2. Yoda1 does not inhibit phosphorylation of EGFR and Vav2.

Figure supplement 3. Yodal does not affect cholesterol localization. 


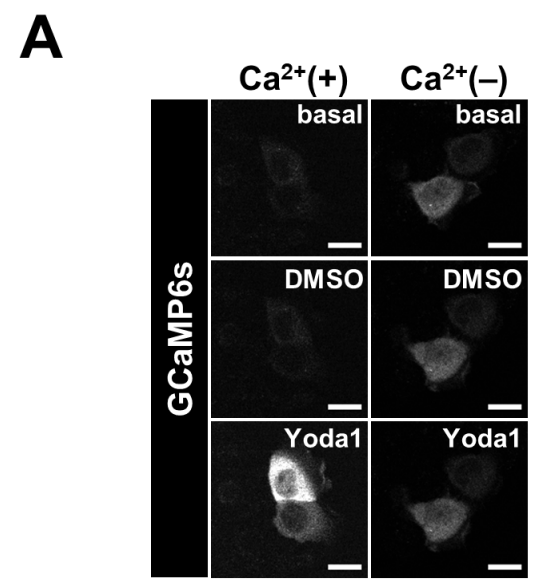

B

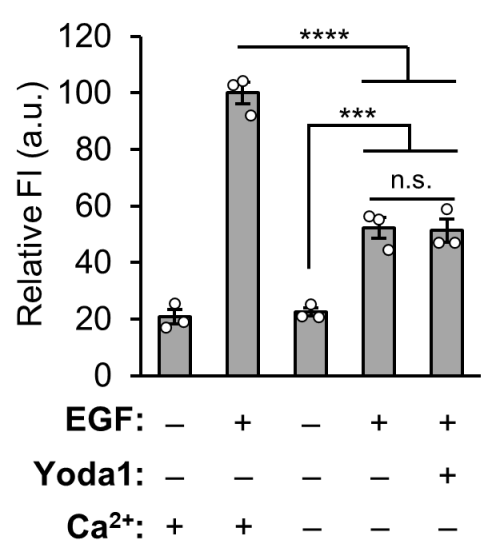

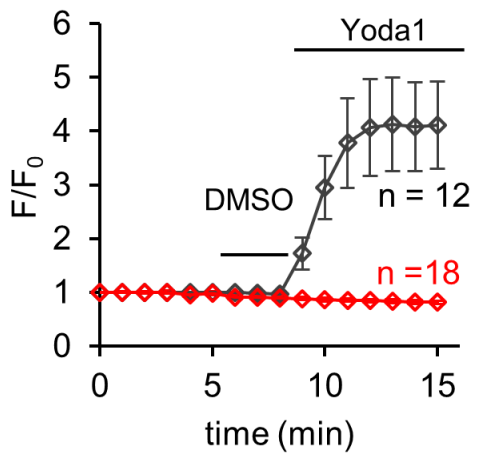

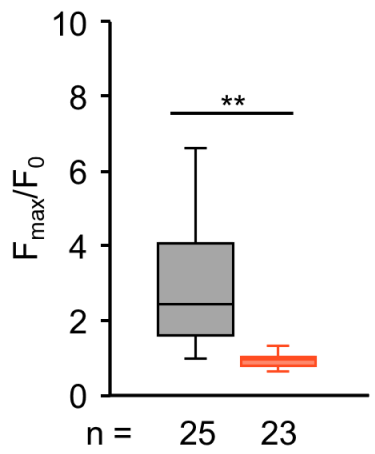

$\square \mathrm{Ca}^{2+}(+)$

C

Figure 3. Macropinocytosis inhibition by Yodal requires extracellular $\mathrm{Ca}^{2+}$ influx. (A) A431

cells were transfected with GCaMP6s and then GCaMP6s fluorescence intensity was recorded every $1 \mathrm{~min}$. DMSO and Yoda1 $(1.5 \mu \mathrm{M})$ were added at 5 and 8 min after start of time-lapse imaging, respectively. Left: Representative images of the GCaMP6s-expressing cells treated DMSO and Yoda1 in the absence or presence of $\mathrm{Ca}^{2+}$ in culture media. Middle: Representative

706 time-course of relative fluorescence intensity of GCaMP6s. Data represent the mean \pm SEM (n

$707=12$ and 18 cells for $\mathrm{Ca}^{2+}(+)$ and $\mathrm{Ca}^{2+}(-)$, respectively). Right: Quantification of maximum

708 Yoda1-induced GCaMP6s intensity increase. Data represent in box plot $(\mathrm{n}=25$ and 23 cells, 709 for $\mathrm{Ca}^{2+}(+)$ and $\mathrm{Ca}^{2+}(-)$, respectively, pooled from two independent experiments). Box and 710 whiskers graph: line, median; box, upper and lower quartiles; whiskers, maxima and minima. 
711 (B) Flow cytometry analysis of TMR-dex70 uptake in $\mathrm{Ca}^{2+}$-free condition. The cells were

712 stimulated with EGF (20 nM) for the uptake of TMR-dex70 in the absence or presence of Yoda1

$713(1.5 \mu \mathrm{M})$ and $\mathrm{Ca}^{2+}$ for $10 \mathrm{~min}$. Data represent the mean $\pm \mathrm{SEM}(\mathrm{n}=3$ independent biological

714 replicates). (C) Confocal microscopy observation of TMR-dex70 uptake in $\mathrm{Ca}^{2+}$-free condition.

715 The cells were treated as $(\mathbf{B})$. The bottom images show enlarged views of the areas outlined by

716 the white squares in the top images. ${ }^{* *}, \mathrm{p}<0.01 ; * * *, \mathrm{p}<0.001 ; * * * *, \mathrm{p}<0.0001$; n.s., not

717 significant (Student's t-test (A) or one-way ANOVA followed by Tukey-Kramer's post hoc test

718 (B)). Scale bars, (A, C top) $20 \mu \mathrm{m}$; (C bottom) $10 \mu \mathrm{m}$. 
A

TRAM-34 + EGF + Yoda1
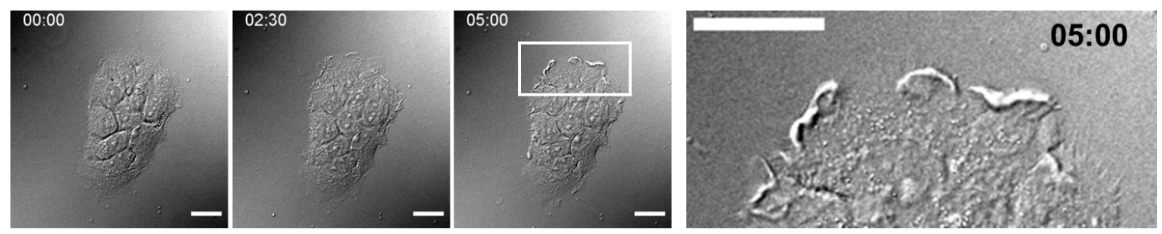

B
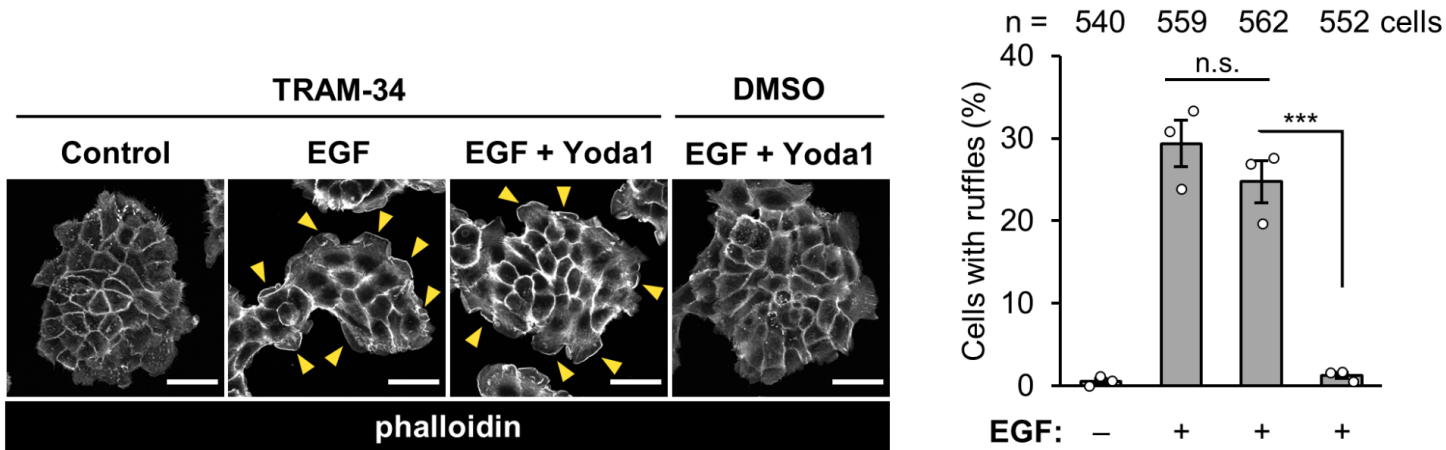

C
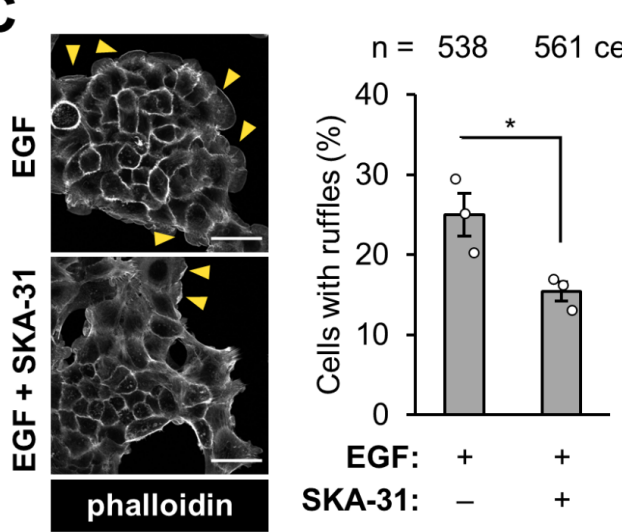

D

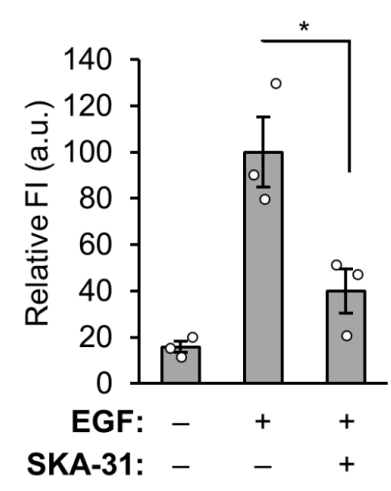

E

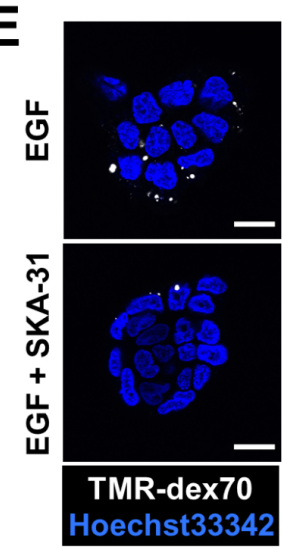

$\mathbf{F}$

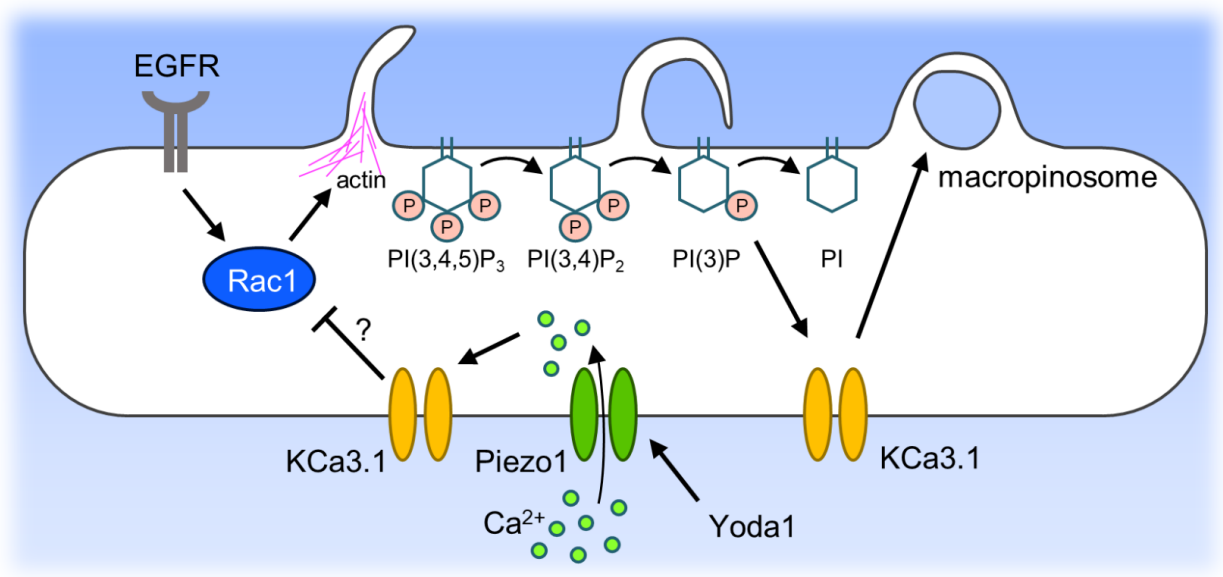

721 Figure 4. Involvement of KCa3.1 in inhibitory effect of Yoda1 on ruffle formation. (A) Live 
cell imaging of EGF-induced membrane ruffles in A431 cells. The cells were pretreated with a KCa3.1 inhibitor TRAM-34 $(10 \mu \mathrm{M})$ for $5 \mathrm{~min}$ and then stimulated with EGF (20 nM) in the presence of Yoda1 $(1.5 \mu \mathrm{M})$. Live cell imaging was started immediately after adding EGF and Yoda1. DIC images at the indicated time points $(0,2.5$ and $5 \mathrm{~min})$ are shown. The right image shows an enlarged view of the area outlined by the white square in the image at 5 min. (B) Factin staining with phalloidin. Left: Representative images of A431 cells pretreated with TRAM-34 $(10 \mu \mathrm{M})$ for $5 \mathrm{~min}$ and then stimulated with EGF $(20 \mathrm{nM})$ in the absence or presence of Yoda1 $(1.5 \mu \mathrm{M})$ for $5 \mathrm{~min}$. Right: Quantification of cells with peripheral ruffles. (C) F-actin staining with phalloidin. Left: Representative images of A431 cells pretreated with KCa3.1 activator SKA-31 $(10 \mu \mathrm{M})$ for $5 \mathrm{~min}$ and then stimulated with EGF (20 $\mathrm{nM})$ for $5 \mathrm{~min}$. Right: Quantification of cells with peripheral ruffles. (D) Flow cytometry analysis of TMR-dex70 uptake. The cells were pretreated with KCa3.1 activator SKA-31 $(10 \mu \mathrm{M})$ for 5 min and then stimulated with EGF $(20 \mathrm{nM})$ for the uptake of TMR-dex70 for $10 \mathrm{~min}$. (E) Confocal microscopy observation of TMR-dex70 uptake. The cells were treated as in (D). (F) Working hypothesis of macropinocytosis inhibition using Yoda1. Yoda1-induced, Piezo1-dependent extracellular $\mathrm{Ca}^{2+}$ influx causes non-proper activation of $\mathrm{KCa} 3.1$, which inhibits Rac1 activation followed by ruffle formation. Data in $(\mathbf{B})$ and $(\mathbf{C})$ represent the mean $\pm \mathrm{SEM}$ (number of total counted cells pooled from three independent experiments: from left to right, (B) 540, 559, 562, and 552; (C) 538 and 561). Data in (D) represent the mean \pm SEM ( $\mathrm{n}=3$ independent biological replicates). ${ }^{*}, \mathrm{p}<0.05 ; * * *, \mathrm{p}<0.001$; n.s., not significant (one-way ANOVA followed by Tukey-Kramer's post hoc test $(\mathbf{B}, \mathbf{D})$ or Student's t-test $(\mathbf{C}))$. Yellow arrowheads in $(\mathbf{B})$ and $(\mathbf{C})$ indicate F-actin positive peripheral membrane ruffling area. Scale bars, $(\mathbf{A}, \mathbf{E})$ $20 \mu \mathrm{m} ;(\mathbf{B}, \mathbf{C}) 50 \mu \mathrm{m}$.

Figure supplement 1. Yoda1 does not induce PI(4,5)P2 depletion. 
A

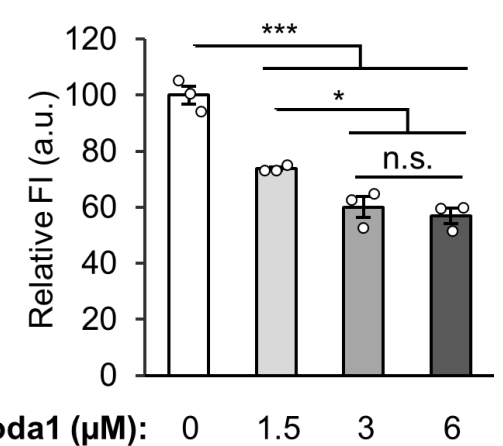

Yoda1 $(\mu \mathrm{M}): 0 \quad 1.5 \quad 3 \quad 6$
B

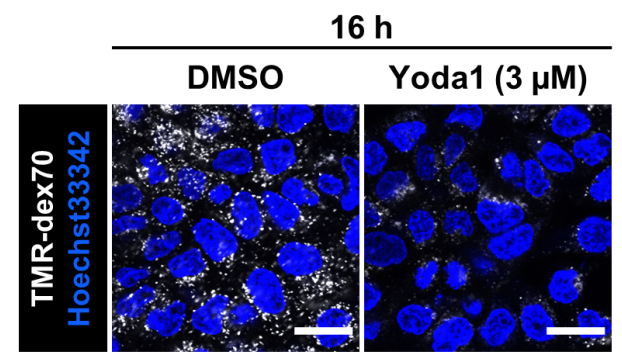

C

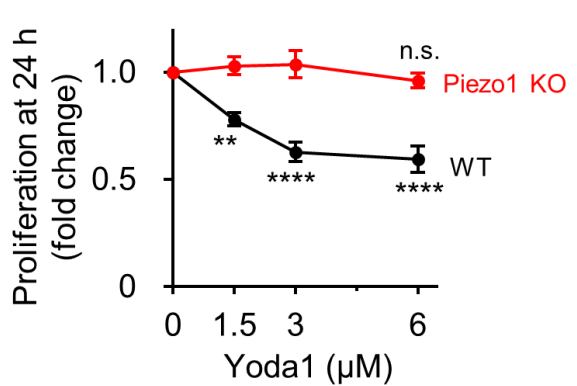

E

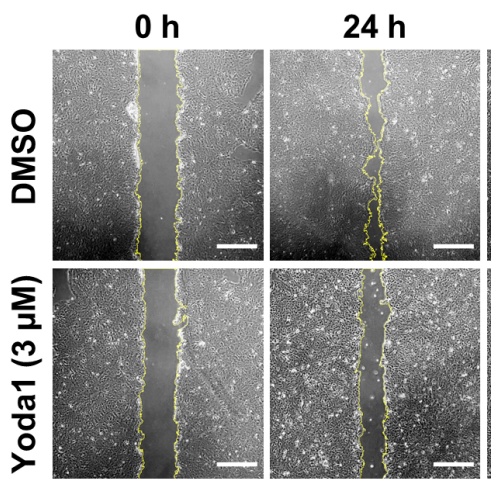

D
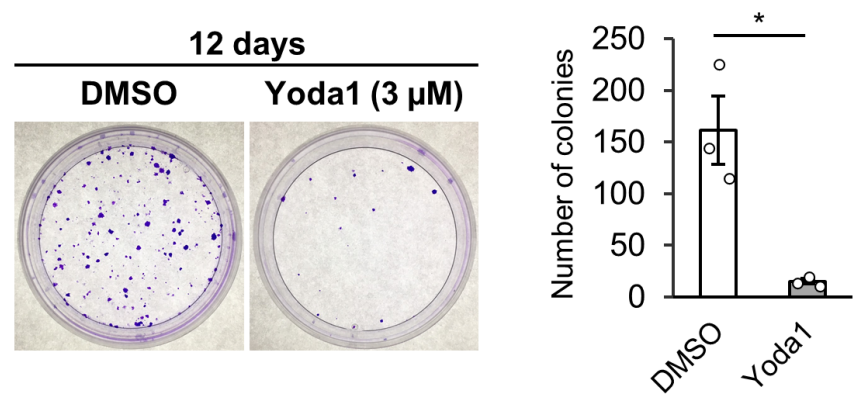

$40 \mathrm{~h}$
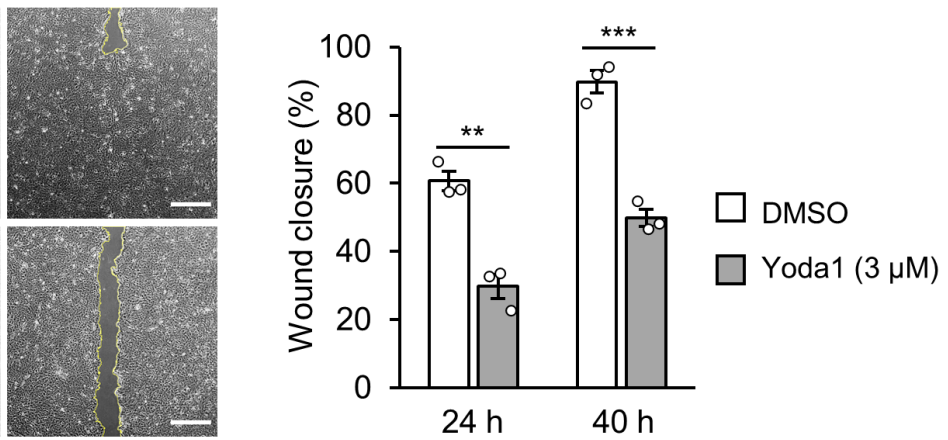

Figure 5. Piezo1 activation inhibits proliferation of A431 cells. (A) Flow cytometry analysis of TMR-dex70 uptake into A431 cells treated with Yoda1 at the indicated concentration for $16 \mathrm{~h}$. (B) Confocal microscopy observation of TMR-dex70 uptake in $16 \mathrm{~h}$. A431 cells were treated TMR-dex70 in the absence or presence of Yodal $(3 \mu \mathrm{M})$ for $16 \mathrm{~h}$. (C) The cell proliferation of

752 A431 cells (WT and Piezo1 KO) in $24 \mathrm{~h}$ was investigated by WST- 8 assay. The cells were

753 treated with Yoda1 at the indicated concentration for $24 \mathrm{~h}$ and then subjected to WST-8 assay.

(D) The cell survival of A431 cells in the presence of Yoda1 was evaluated by colony formation 
assay. The cells in $35 \mathrm{~mm}$ dishes were treated with DMSO or Yoda1 ( $3 \mu \mathrm{M})$ for 12 days, fixed, stained with $0.2 \%$ crystal violet. Left: Representative images. Right: Quantification of the number of visible colonies. Culture medium was changed every 3 days. (E) The migration and

758 proliferation of A431 cells in the presence of Yodal was measured by wound healing assay. A 759 confluent monolayer of A431 cells was scratched and then treated with DMSO or Yoda1 (3 $\mu \mathrm{M})$ for $40 \mathrm{~h}$. Left: Representative images. Right: Quantification of the wound closure. Yellow lines indicate the boundary between cells and the scratched area. Data represent the mean \pm SEM ( $\mathrm{n}=3$ independent biological replicates) in (A), (C), (D) and (E). *, $\mathrm{p}<0.05 ;{ }^{* *}, \mathrm{p}<$ $0.01 ; * * *, \mathrm{p}<0.001 ; * * * *, \mathrm{p}<0.0001 ;$ n.s., not significant (one-way ANOVA followed by

764 Tukey-Kramer's post hoc test (A) or one-way ANOVA followed by Dunnett's post hoc test (C) or Student's t-test (D, E)). Scale bars, (B) $25 \mu \mathrm{m}$; (E) $500 \mu \mathrm{m}$.

Figure supplement 1. Inhibition of dextran uptake in $16 \mathrm{~h}$ by EIPA and Yoda1.

767 Figure supplement 2. Yoda1 inhibits constitutive macropinocytosis and proliferation of 768 HT1080 cells. 


\section{References}

770 1. Swanson, J.A. \& Watts, C. Macropinocytosis. Trends in Cell Biology 5, 424-428 (1995).

2. Swanson, J.A. Shaping cups into phagosomes and macropinosomes. Nature Reviews Molecular Cell Biology 9, 639-649 (2008).

3. Lim, J.P. \& Gleeson, P.A. Macropinocytosis: an endocytic pathway for internalising large gulps. Immunology and Cell Biology 89, 836-843 (2011).

4. $\quad$ Conner, S.D. \& Schmid, S.L. Regulated portals of entry into the cell. Nature 422, 37 44 (2003).

5. Commisso, C. et al. Macropinocytosis of protein is an amino acid supply route in Rastransformed cells. Nature 497, 633-+ (2013).

6. Yoshida, S., Pacitto, R., Inoki, K. \& Swanson, J. Macropinocytosis, mTORC1 and cellular growth control. Cellular and Molecular Life Sciences 75, 1227-1239 (2018).

7. Song, S.J., Zhang, Y.N., Ding, T.T., Ji, N. \& Zhao, H. The Dual Role of Macropinocytosis in Cancers: Promoting Growth and Inducing Methuosis to Participate in Anticancer Therapies as Targets. Frontiers in Oncology 10(2021).

8. Desai, A.S., Hunter, M.R. \& Kapustin, A.N. Using macropinocytosis for intracellular delivery of therapeutic nucleic acids to tumour cells. Philosophical Transactions of the Royal Society B-Biological Sciences 374(2019).

9. Futaki, S., Arafiles, J.V.V. \& Hirose, H. Peptide-assisted Intracellular Delivery of Biomacromolecules. Chemistry Letters 49, 1088-1094 (2020).

10. Doodnauth, S.A., Grinstein, S. \& Maxson, M.E. Constitutive and stimulated macropinocytosis in macrophages: roles in immunity and in the pathogenesis of atherosclerosis. Philosophical Transactions of the Royal Society B-Biological Sciences 374(2019).

11. Canton, J. et al. Calcium-sensing receptors signal constitutive macropinocytosis and facilitate the uptake of NOD2 ligands in macrophages. Nature Communications 7(2016).

12. Swanson, J.A. Phorbol Esters Stimulate Macropinocytosis and Solute Flow through Macrophages. Journal of Cell Science 94, 135-142 (1989).

13. Tanaka, G. et al. CXCR4 Stimulates Macropinocytosis: Implications for Cellular Uptake of Arginine-Rich Cell-Penetrating Peptides and HIV. Chemistry \& Biology 19, 1437-1446 (2012).

14. Pacitto, R., Gaeta, I., Swanson, J.A. \& Yoshida, S. CXCL12-induced macropinocytosis modulates two distinct pathways to activate mTORC1 in macrophages. Journal of Leukocyte Biology 101, 683-692 (2017).

15. Yoshida, S., Pacitto, R., Sesi, C., Kotula, L. \& Swanson, J.A. Dorsal ruffles enhance activation of Akt by growth factors. Journal of Cell Science 131(2018).

16. Egami, Y., Taguchi, T., Maekawa, M., Arai, H. \& Araki, N. Small GTPases and 
806

807

808

809

810

811

812

813

814

815

816

817

818

819

820

821

822

823

824

825

826

827

828

829

830

831

832

833

834

835

836

837

838

839

840

841

842

843

phosphoinositides in the regulatory mechanisms of macropinosome formation and maturation. Frontiers in Physiology 5(2014).

17. Loh, J. et al. An acute decrease in plasma membrane tension induces macropinocytosis via PLD2 activation. Journal of Cell Science 132(2019).

18. Diz-Munoz, A., Fletcher, D.A. \& Weiner, O.D. Use the force: membrane tension as an organizer of cell shape and motility. Trends in Cell Biology 23, 47-53 (2013).

19. Raucher, D. \& Sheetz, M.P. Cell spreading and lamellipodial extension rate is regulated by membrane tension. Journal of Cell Biology 148, 127-136 (2000).

20. Martino, F., Perestrelo, A.R., Vinarsky, V., Pagliari, S. \& Forte, G. Cellular Mechanotransduction: From Tension to Function. Frontiers in Physiology 9(2018).

21. Cox, C.D., Bavi, N. \& Martinac, B. Biophysical Principles of Ion-Channel-Mediated Mechanosensory Transduction. Cell Reports 29, 1-12 (2019).

22. Le Roux, A.L., Quiroga, X., Walani, N., Arroyo, M. \& Roca-Cusachs, P. The plasma membrane as a mechanochemical transducer. Philosophical Transactions of the Royal Society B-Biological Sciences 374(2019).

23. Coste, B. et al. Piezo1 and Piezo2 Are Essential Components of Distinct Mechanically Activated Cation Channels. Science 330, 55-60 (2010).

24. Sun, W.J. et al. The mechanosensitive Piezo1 channel is required for bone formation. Elife 8(2019).

25. Liu, C.S.C. et al. Cutting Edge: Piezo1 Mechanosensors Optimize Human T Cell Activation. Journal of Immunology 200, 1255-1260 (2018).

26. Tsuchiya, M. et al. Cell surface flip-flop of phosphatidylserine is critical for PIEZO1mediated myotube formation. Nature Communications 9(2018).

27. Wang, S.P. et al. Endothelial cation channel PIEZO1 controls blood pressure by mediating flow-induced ATP release. Journal of Clinical Investigation 126, 4527-4536 (2016).

28. Cahalan, S.M. et al. Piezo1 links mechanical forces to red blood cell volume. Elife 4(2015).

29. Nonomura, K. et al. Mechanically activated ion channel PIEZO1 is required for lymphatic valve formation. Proceedings of the National Academy of Sciences of the United States of America 115, 12817-12822 (2018).

30. Zhang, G.D., Li, X.F., Wu, L. \& Qin, Y.X. Piezo1 channel activation in response to mechanobiological acoustic radiation force in osteoblastic cells. Bone Research 9(2021).

31. Syeda, R. et al. Chemical activation of the mechanotransduction channel Piezo1. Elife 4(2015).

32. Botello-Smith, W.M. et al. A mechanism for the activation of the mechanosensitive Piezo1 channel by the small molecule Yoda1. Nature Communications 10(2019).

33. Maekawa, M. et al. Sequential breakdown of 3-phosphorylated phosphoinositides is 
essential for the completion of macropinocytosis. Proceedings of the National Academy of Sciences of the United States of America 111, E978-E987 (2014).

34. Ran, F.A. et al. Genome engineering using the CRISPR-Cas9 system. Nature Protocols 8, 2281-2308 (2013).

35. Chen, T.W. et al. Ultrasensitive fluorescent proteins for imaging neuronal activity. Nature 499, 295-300 (2013).

36. Bloomfield, G. \& Kay, R.R. Uses and abuses of macropinocytosis. Journal of Cell Science 129, 2697-2705 (2016).

37. Isogai, T. et al. Initiation of lamellipodia and ruffles involves cooperation between mDia1 and the Arp2/3 complex. Journal of Cell Science 128, 3796-3810 (2015).

38. Ridley, A.J., Paterson, H.F., Johnston, C.L., Diekmann, D. \& Hall, A. The Small GtpBinding Protein Rac Regulates Growth-Factor Induced Membrane Ruffling. Cell 70, 401-410 (1992).

39. Koivusalo, M. et al. Amiloride inhibits macropinocytosis by lowering submembranous $\mathrm{pH}$ and preventing Rac1 and Cdc42 signaling. Journal of Cell Biology 188, 547-563 (2010).

40. Grimmer, S., van Deurs, B. \& Sandvig, K. Membrane ruffling and macropinocytosis in A431 cells require cholesterol. Journal of Cell Science 115, 2953-2962 (2002).

41. Maekawa, M. \& Fairn, G.D. Complementary probes reveal that phosphatidylserine is required for the proper transbilayer distribution of cholesterol. Journal of Cell Science 128, 1422-1433 (2015).

42. Mendoza, S.A. et al. TRPV4-mediated endothelial $\mathrm{Ca} 2+$ influx and vasodilation in response to shear stress. American Journal of Physiology-Heart and Circulatory Physiology 298, H466-H476 (2010).

43. Swanson, J.A. \& Yoshida, S. Macropinosomes as units of signal transduction. Philosophical Transactions of the Royal Society B-Biological Sciences 374(2019).

44. Srivastava, S. et al. The phosphatidylinositol 3-phosphate phosphatase myotubularinrelated protein 6 (MTMR6) is a negative regulator of the $\mathrm{Ca} 2+$-Activated $\mathrm{K}+$ channel K(Ca)3.1. Molecular and Cellular Biology 25, 3630-3638 (2005).

45. Zoncu, R. et al. Loss of endocytic clathrin-coated pits upon acute depletion of phosphatidylinositol 4,5-bisphosphate. Proceedings of the National Academy of Sciences of the United States of America 104, 3793-3798 (2007).

46. Maekawa, M. \& Fairn, G.D. Molecular probes to visualize the location, organization and dynamics of lipids. Journal of Cell Science 127, 4801-4812 (2014).

47. Sankaranarayanan, A. et al. Naphtho[1,2-d]thiazol-2-ylamine (SKA-31), a New Activator of $\mathrm{KCa} 2$ and $\mathrm{KCa} 3.1$ Potassium Channels, Potentiates the EndotheliumDerived Hyperpolarizing Factor Response and Lowers Blood Pressure. Molecular Pharmacology 75, 281-295 (2009). 
882

883

884

885

886

887

888

889

890

891

892

893

894

895

896

897

898

899

900

901

902

903

904

905

906

907

908

909

910

911

912

913

914

915

916

917

918

919

48. Recouvreux, M.V. \& Commisso, C. Macropinocytosis: A Metabolic Adaptation to nutrient Stress in Cancer. Frontiers in Endocrinology 8(2017).

49. Zhang, Y.J. \& Commisso, C. Macropinocytosis in Cancer: A Complex Signaling Network. Trends in Cancer 5, 332-334 (2019).

50. Zhang, J.L. et al. PIEZO1 functions as a potential oncogene by promoting cell proliferation and migration in gastric carcinogenesis. Molecular Carcinogenesis 57, 1144-1155 (2018).

51. Palm, W. Metabolic functions of macropinocytosis. Philosophical Transactions of the Royal Society B-Biological Sciences 374(2019).

52. Stow, J.L., Hung, Y. \& Wall, A.A. Macropinocytosis: Insights from immunology and cancer. Current Opinion in Cell Biology 65, 131-140 (2020).

53. Ivanov, A.I. Pharmacological Inhibition of Endocytic Pathways: Is It Specific Enough to Be Useful? Methods in Molecular Biology 440, 15-33 (2008).

54. Lin, H.P. et al. Identification of novel macropinocytosis inhibitors using a rational screen of Food and Drug Administration-approved drugs. British Journal of Pharmacology 175, 3640-3655 (2018).

55. Fujimoto, L.M., Roth, R., Heuser, J.E. \& Schmid, S.L. Actin assembly plays a variable, but not obligatory role in receptor-mediated endocytosis in mammalian cells. Traffic $\mathbf{1}$, 161-171 (2000).

56. Araki, N., Johnson, M.T. \& Swanson, J.A. A role for phosphoinositide 3-kinase in the completion of macropinocytosis and phagocytosis by macrophages. Journal of Cell Biology 135, 1249-1260 (1996).

57. Liu, Y.S. et al. Wortmannin, a widely used phosphoinositide 3-kinase inhibitor, also potently inhibits mammalian polo-like kinase. Chemistry \& Biology 12, 99-107 (2005).

58. Dai, X.Q. et al. Inhibition of TRPP3 channel by amiloride and analogs. Molecular Pharmacology 72, 1576-1585 (2007).

59. dela Paz, N.G. \& Frangos, J.A. Yoda1-induced phosphorylation of the Akt and ERK1/2 does not require Piezo1 activation. Faseb Journal 32(2018).

60. Sun, Y.H. et al. The function of Piezol in colon cancer metastasis and its potential regulatory mechanism. Journal of Cancer Research and Clinical Oncology 146, 11391152 (2020).

61. Han, Y. et al. Mechanosensitive ion channel Piezol promotes prostate cancer development through the activation of the Akt/mTOR pathway and acceleration of cell cycle. International Journal of Oncology 55, 629-644 (2019).

62. Wu, J., Lewis, A.H. \& Grandl, J. Touch, Tension, and Transduction - The Function and Regulation of Piezo Ion Channels. Trends in Biochemical Sciences 42, 57-71 (2017).

63. Tsai, F.C. et al. A polarized $\mathrm{Ca} 2+$, diacylglycerol and STIM1 signalling system regulates directed cell migration. Nature Cell Biology 16, 133-144 (2014). 
A

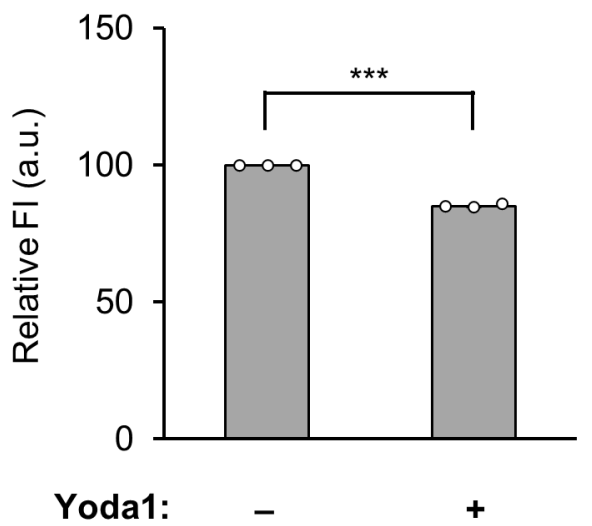

B

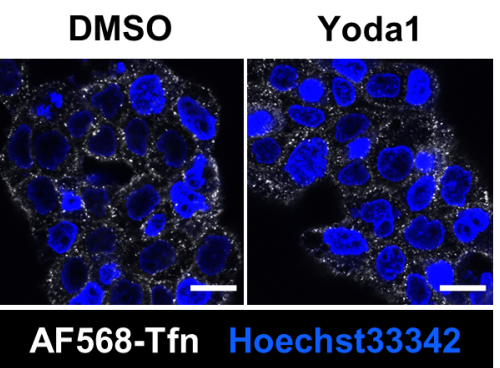

AF568-Tfn Hoechst33342

923 Figure 1-figure supplement 1. Tfn uptake in the absence or presence of Yoda1. (A) Flow 924 cytometry analysis of AF568-Tfn uptake into A431 cells. The cells were incubated with AF568$925 \mathrm{Tfn}(20 \mu \mathrm{g} / \mathrm{mL})$ in the absence or presence of Yodal $(1.5 \mu \mathrm{M})$ for $10 \mathrm{~min}$. Data represent the 926 mean \pm SEM ( $\mathrm{n}=3$ independent biological replicates). (B) Confocal microscopy observation 927 of AF568-Tfn uptake. A431 cells were treated as (A). Scale bars, $20 \mu \mathrm{m}$. 

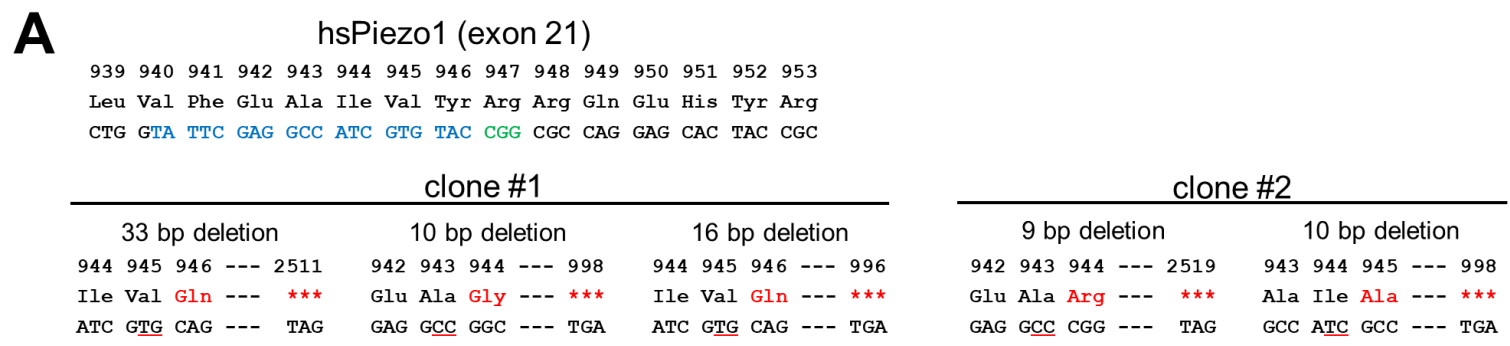

\section{B}

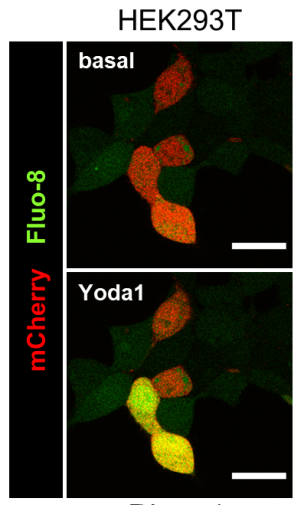

Piezo1 full length

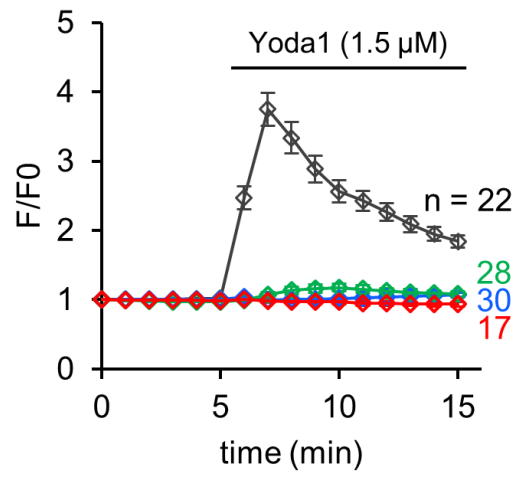

full length empty vector

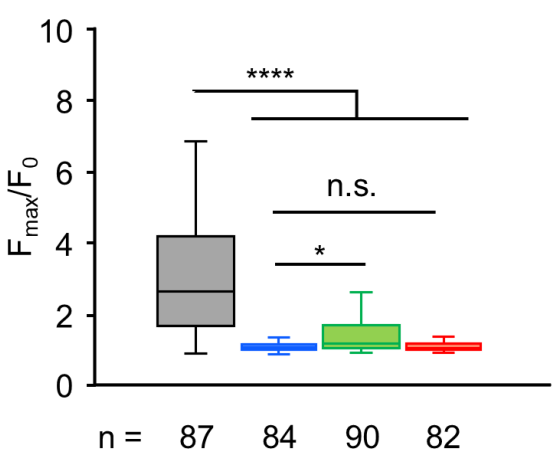

$\Delta 946-956$

930 Figure 1-figure supplement 2. Supporting data for characterization of Piezo1 knockout A431

931

932

933

934

935

936

937

941

cells. (A) CRISPR/Cas9 target site of the Piezo1 gene. The guide sequence and the protospacer adjacent motif are showed in blue and green characters, respectively. CRISPR/Cas9-mediated deletions are showed in red characters. (B) HEK293T cells were transfected with indicated vector, loaded with Fluo-8 AM $(5 \mu \mathrm{M})$ and then Fluo-8 fluorescence intensity was recorded every $1 \mathrm{~min}$. Transient gene expression was confirmed by IRES2 dependent co-expression of mCherry. Yoda1 $(1.5 \mu \mathrm{M})$ was added 5 min after start of time-lapse imaging. Left: Representative images before and after Yoda1 addition. Middle: Representative time-course of relative fluorescence intensity of Fluo-8 AM. Data represent the mean \pm SEM. Right: Quantification of maximum Yoda1-induced GCaMP6s intensity increase. ( $\mathrm{n} \geq 82$ cells for each condition pooled from three independent experiments.) Box and whiskers graph: line, median; box, upper and lower quartiles; whiskers, maxima and minima; circle plots, outliers. Scale bars, 
bioRxiv preprint doi: https://doi.org/10.1101/2021.05.14.444123; this version posted May 14, 2021. The copyright holder for this preprint (which was not certified by peer review) is the author/funder. All rights reserved. No reuse allowed without permission.

$94225 \mu \mathrm{m} . * * * *, \mathrm{P}<0.0001 ; *$ * $\mathrm{P}<0.05$; n.s., not signficant (one-way ANOVA followed by Tukey-

943 Kramer's post hoc test). 
Figure 1-figure supplement 3

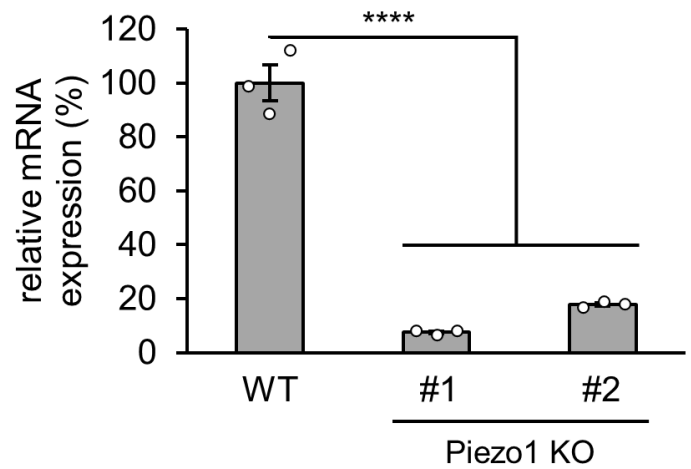

946 Figure 1-figure supplement 3. Piezo1 gene expression examined by qPCR. Expression of 947 Piezo1 in A431 cells (WT, KO clone \#1, 2). Piezo1 mRNA expression level was determined by 948 qPCR. The GAPDH shows relative mRNA expression level of Piezo1 in each condition. Data 949 represent mean $\pm \mathrm{SD}$ of triplicate samples from one of two independent experiments. ****, $\mathrm{P}$ $950<0.0001$ (one-way ANOVA followed by Dunnett's post hoc test). 
A

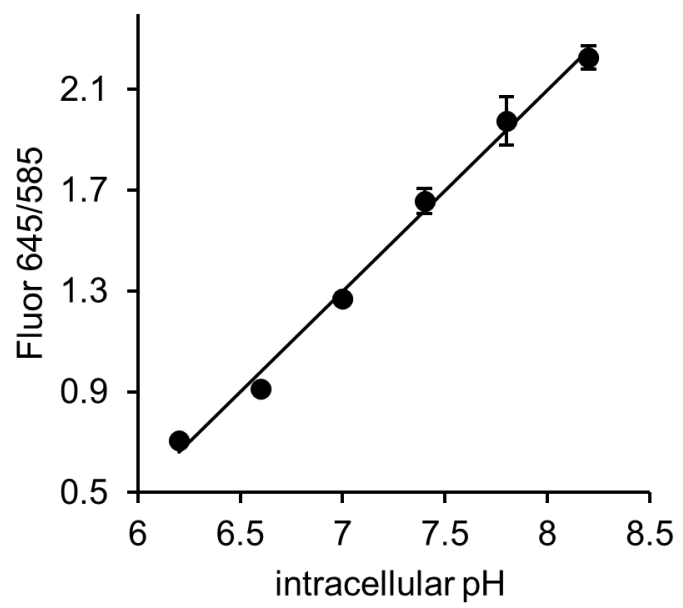

B

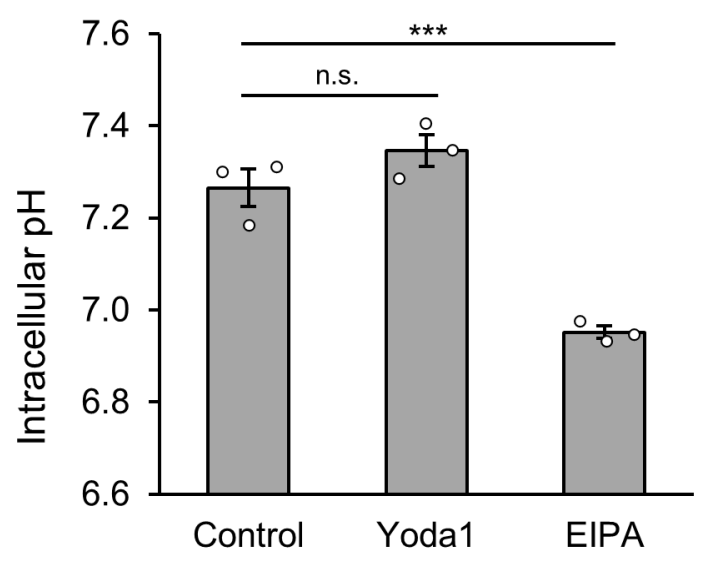

952

Figure 2-figure supplement 1. Yoda1 does not change intracellular $\mathrm{pH}$. (A) Relationship between fluorescence intensity ratio $(645 / 585 \mathrm{~nm})$ of SNARF-5F AM and intracellular $\mathrm{pH}$. Fluorescence intensity ratio $(645 / 585 \mathrm{~nm})$ of SNARF-5F AM was plotted against respective $\mathrm{pH}$ and linear regression analysis was performed to obtain the calibration curve. Data represent mean \pm SEM ( $\mathrm{n}=150$ cells for each condition from three independent experiments $)$. (B) Intracellular pH measurement using SNARF-5F AM in starved A431 cells treated either with Yoda1 $(1.5 \mu \mathrm{M})$ for $10 \mathrm{~min}$ or EIPA $(25 \mu \mathrm{M})$ for $30 \mathrm{~min}$ in D-MEM(-). EIPA was used as a positive control to reduce intracellular $\mathrm{pH}$. The intracellular $\mathrm{pH}$ value was obtained from the calibration curve in $(\mathbf{A})$. Data represent mean \pm SEM $(n=150$ cells for each condition from three independent experiments). ${ }^{* * *}, \mathrm{P}<0.001 ;$ n.s., not significant (one-way ANOVA followed by Dunnett's post hoc test). 
Figure 2-figure supplement 2

A

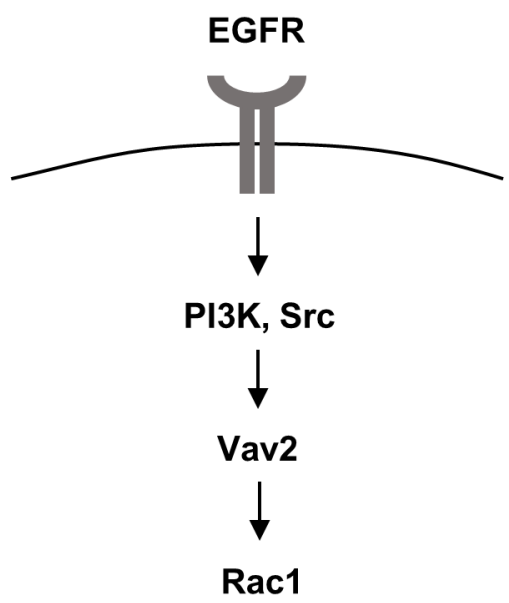

B

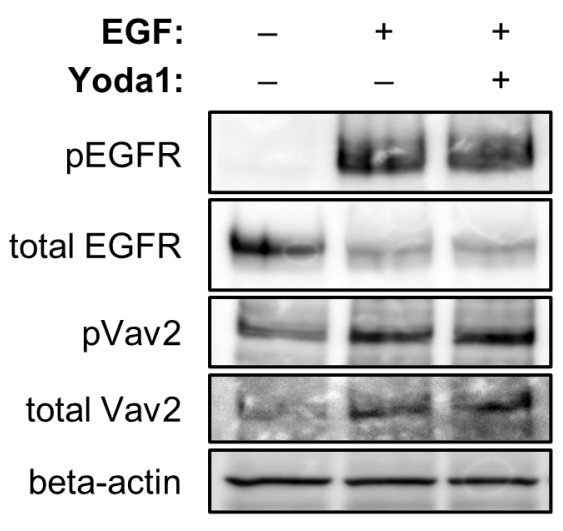

965

966 Figure 2-figure supplement 2. Yoda1 does not inhibit phosphorylation of EGFR and Vav2.

967 (A) Schematic diagram showing the pathway of Rac1 activation by EGF. (B) Western blot analysis. A431 cells were treated with EGF $(20 \mathrm{nM})$ and Yoda1 $(1.5 \mu \mathrm{M})$ for $5 \mathrm{~min}$, and total cell lysates were analyzed by performing SDS-PAGE followed by western blot. The experiments were performed at least twice with similar results. 


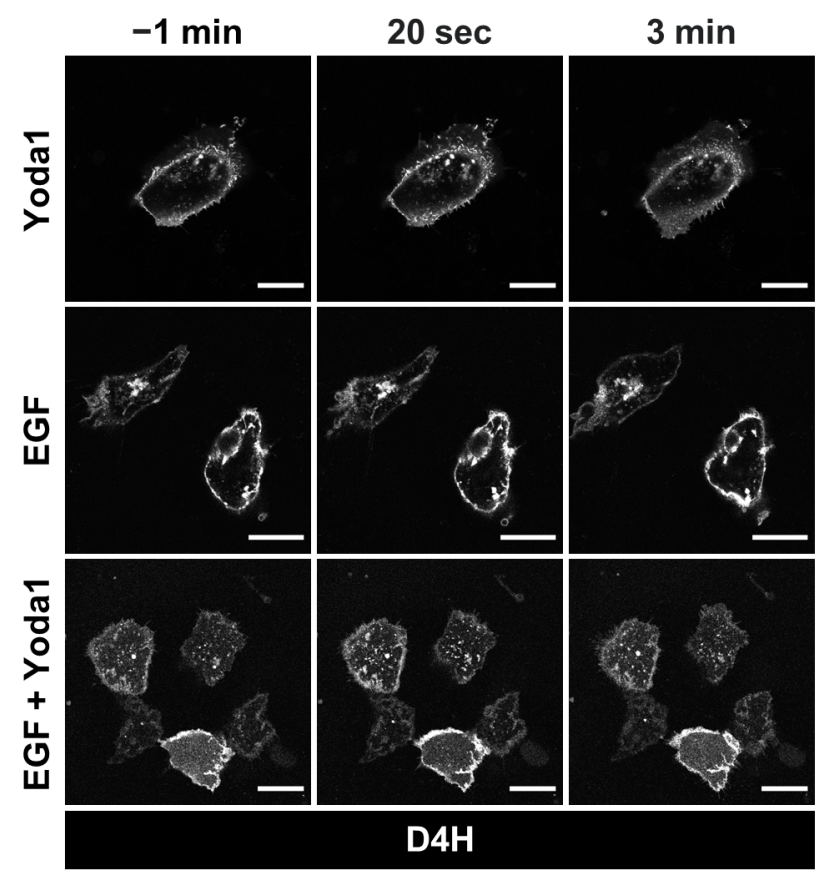

972

973 Figure 2-figure supplement 3. Yoda1 does not affect cholesterol localization. A431 cells

974 were transfected with mCherry-tagged $\mathrm{D} 4 \mathrm{H}$, a specific probe for cholesterol, serum-starved for

$9754 \mathrm{~h}$, and then mCherry-D4H fluorescence images were acquired every $20 \mathrm{sec}$. The time of EGF

$976(20 \mathrm{nM})$ and Yoda1 $(1.5 \mu \mathrm{M})$ addition was defined as time $=0$. Images at indicated time points

977 (1 min before adding reagents and $20 \mathrm{sec}, 3 \mathrm{~min}$ after addition) are shown. Scale bars, $20 \mu \mathrm{m}$. 

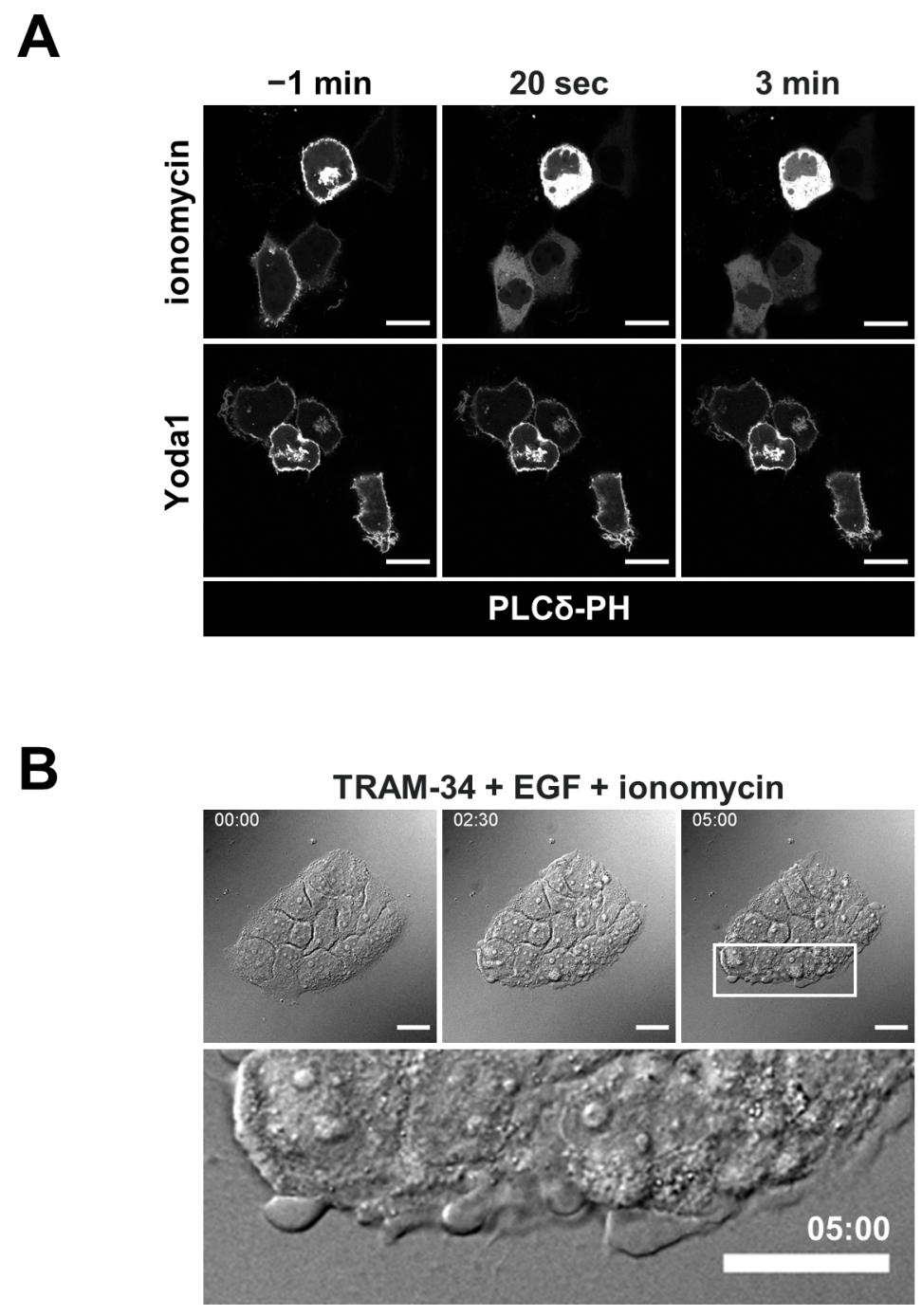

980 Figure 4-figure supplement 1. Yoda1 does not induce PI(4,5)P2 depletion. (A) A431 cells 981 were transfected with GFP-tagged PLC $\delta$-PH, a specific probe for PI(4,5)P2, and then GFP982 PLC-PH fluorescence images were acquired every $20 \mathrm{~s}$. The time of ionomycin $(5 \mu \mathrm{M})$ or 983 Yoda1 $(1.5 \mu \mathrm{M})$ addition was defined as time $=0$. Images at indicated time points $(1$ min before 984 adding reagents, $20 \mathrm{~s}$ and $3 \mathrm{~min}$ after addition) are shown. (B) Live imaging shows that 985 ionomycin inhibits EGF-induced ruffle also in the presence of TRAM-34. A431 cells were 986 pretreated with a KCa3.1 inhibitor TRAM-34 $(10 \mu \mathrm{M})$ for 5 min and then stimulated with EGF $987(20 \mathrm{nM})$ in the presence of ionomycin $(5 \mu \mathrm{M})$. Time-lapse imaging was started immediately 
bioRxiv preprint doi: https://doi.org/10.1101/2021.05.14.444123; this version posted May 14, 2021. The copyright holder for this preprint (which was not certified by peer review) is the author/funder. All rights reserved. No reuse allowed without permission.

988 after adding EGF and ionomycin. DIC images at the indicated time points $(0 \mathrm{~min}, 2.5 \mathrm{~min}$ and

$9895 \mathrm{~min}$ ) are shown. The bottom image shows an enlarged view of the area outlined by the white

990 square in the image at $5 \mathrm{~min}$. Scale bars, $(\mathbf{A}, \mathbf{B}) 20 \mu \mathrm{m}$. 
A

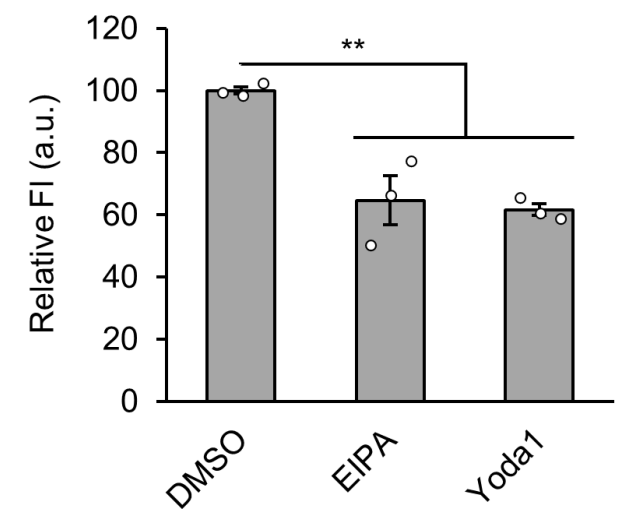

B

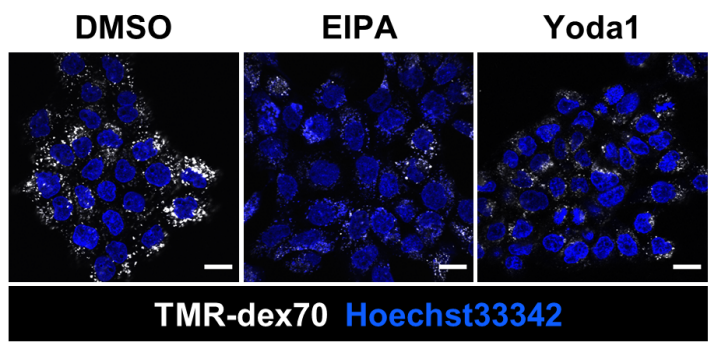

992

Figure 5-figure supplement 1. Inhibition of dextran uptake in $16 \mathrm{~h}$ by EIPA and Yoda1. (A)

994 Flow cytometry analysis of TMR-dex70 uptake into A431 cells. The cells were treated with

995 TMR-dex70 $(0.5 \mathrm{mg} / \mathrm{mL})$ in the presence of DMSO as vehicle control, EIPA $(20 \mu \mathrm{M})$ or Yoda1

$996(1.5 \mu \mathrm{M})$ in serum-containing medium for $16 \mathrm{~h}$. (B) Confocal microscopy observation of TMR-

997 dex70 uptake in $16 \mathrm{~h}$. A431 cells were treated as (A). Data represent the mean \pm SEM $(\mathrm{n}=3$

998 independent biological replicates) in (A). ${ }^{* *}, \mathrm{p}<0.01$ (one-way ANOVA followed by Dunnett's

999 post hoc test). Scale bars, $20 \mu \mathrm{m}$. 
A

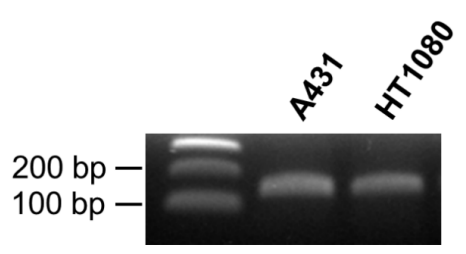

B
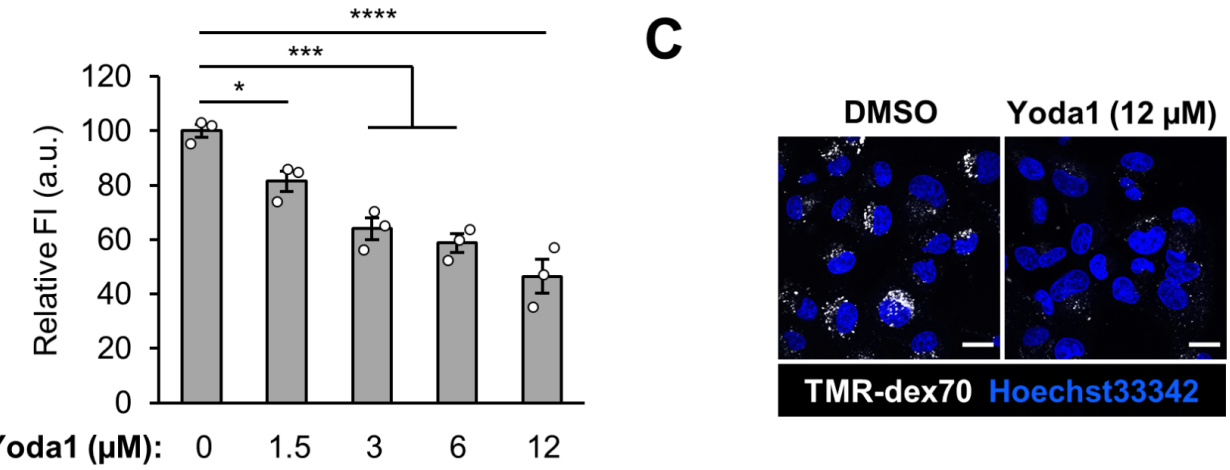

D
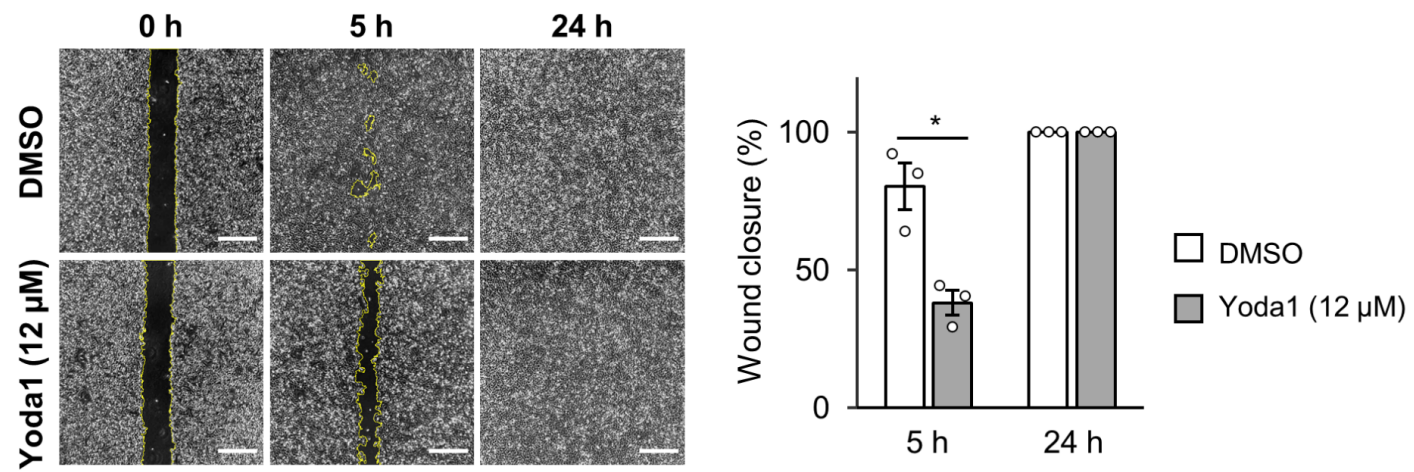

Figure 5-figure supplement 2. Yoda1 inhibits constitutive macropinocytosis and proliferation of HT1080 cells. (A) Expression of Piezo1 in HT1080 cells. mRNA expression of Piezo1 was confirmed by RT-PCR. (B) Flow cytometry analysis of TMR-dex70 uptake into A431 cells. The cells were incubated with TMR-dex70 $(0.5 \mathrm{mg} / \mathrm{mL})$ in the absence or presence of Yodal at indicated concentration for $4 \mathrm{~h}$. (C) Microscopy observation of TMR-dex70 uptake. The cells were incubated with TMR-dex70 $(0.5 \mathrm{mg} / \mathrm{mL})$ in the absence or presence of Yoda1 $(12 \mu \mathrm{M})$ for $4 \mathrm{~h}$. (D) The cell migration and proliferation of HT1080 cells in the presence of Yoda1 were measured by wound healing assay. A confluent monolayer of HT1080 cells was scratched, treated with DMSO or Yoda1 $(12 \mu \mathrm{M})$ for $24 \mathrm{~h}$. Left: Representative images. Right: 
bioRxiv preprint doi: https://doi.org/10.1101/2021.05.14.444123; this version posted May 14, 2021. The copyright holder for this preprint (which was not certified by peer review) is the author/funder. All rights reserved. No reuse allowed without permission.

1011 Quantification of the wound closure. *, p < 0.05; ***, p $<0.001$; ****, p $<0.0001$ (one-way

1012 ANOVA followed by Dunnett's post hoc test (C)). Scale bars, (C) $20 \mu \mathrm{m}$; (D right) $500 \mu \mathrm{m}$. 
Videos

1014 Video 1. EGF induces peripheral ruffle formation. Differential interference contrast (DIC)

1015

1016

1017

1018

1019

1020

1021

1022

1023

1024

1025

1026

1027

1028

1029

1030

1031 movie of A431 cells treated with EGF (20 nM). Time-lapse imaging was started immediately after adding EGF, and frames were acquired every $10 \mathrm{~s}$. Video was sped up 100× (ten frames per second) over real time. Scale bar, $20 \mu \mathrm{m}$.

Video 2. Yodal inhibits ruffle peripheral formation. Differential interference contrast (DIC) movie of A431 cells treated with EGF $(20 \mathrm{nM})$ and Yoda1 $(1.5 \mu \mathrm{M})$. Time-lapse imaging was started immediately after adding EGF and Yoda1, and frames were acquired every $10 \mathrm{~s}$. Video was sped up $100 \times$ (ten frames per second) over real time. Scale bar, $20 \mu \mathrm{m}$.

Video 3. TRAM-34 recovers peripheral formation in the presence of Yoda1. Differential interference contrast (DIC) movie of A431 cells pretreated with TRAM-34 (10 $\mu \mathrm{M})$ for 5 min and then treated with EGF $(20 \mathrm{nM})$ and Yodal $(1.5 \mu \mathrm{M})$. Time-lapse imaging was started immediately after adding EGF and Yoda1, and frames were acquired every $10 \mathrm{~s}$. Video was sped up $100 \times$ (ten frames per second) over real time. Scale bar, $20 \mu \mathrm{m}$.

Video 4. TRAM-34 does not recover peripheral formation in the presence of ionomycin. Differential interference contrast (DIC) movie of A431 cells pretreated with TRAM-34 $(10 \mu \mathrm{M})$ for $5 \mathrm{~min}$ and then treated with EGF $(20 \mathrm{nM})$ and ionomycin $(5 \mu \mathrm{M})$. Time-lapse imaging was started immediately after adding EGF and ionomycin, and frames were acquired every $10 \mathrm{~s}$. Video was sped up 100× (ten frames per second) over real time. Scale bar, $20 \mu \mathrm{m}$. 